\title{
COLD DUST IN HOT REGIONS
}

\author{
Gopika Sreenilayam ${ }^{1}$, Michel Fich ${ }^{1}$, Peter Ade ${ }^{2}$, Dan Bintley $^{3}$, Ed Chapin ${ }^{3,4,5}$, Antonio Chrysostomou ${ }^{3}$, \\ James S. Dunlop ${ }^{6}$, Andy GibB ${ }^{5}$, Jane S. Greaves ${ }^{7}$, Mark Halpern ${ }^{5}$, Wayne S. Holland ${ }^{6,8}$, Rob Ivison $^{6,8}$, \\ Tim Jenness ${ }^{3,9}$, IAN Robson ${ }^{8}$, And Douglas ScotT ${ }^{5}$ \\ ${ }^{1}$ Department of Physics and Astronomy, University of Waterloo, Waterloo, Ontario, N2L 3G1, Canada \\ ${ }^{2}$ Cardiff School of Physics and Astronomy, Cardiff University, Queen's Building, The Parade, Cardiff CF24 3AA, UK \\ ${ }^{3}$ Joint Astronomy Centre, 660 N. A'ohoku Place, University Park, Hilo, HI 96720, USA \\ ${ }^{4}$ XMM SOC, ESAC, Apartado 78, E-28691 Villanueva de la Canada, Madrid, Spain \\ ${ }^{5}$ Department of Physics and Astronomy, University of British Columbia, 6224 Agricultural Road, Vancouver BC V6T 1Z1, Canada \\ ${ }^{6}$ Institute for Astronomy, University of Edinburgh, Royal Observatory, Blackford Hill, Edinburgh EH9 3HJ, UK \\ ${ }^{7}$ School of Physics and Astronomy, University of St Andrews, North Haugh, St Andrews, Fife KY16 9SS, UK \\ ${ }^{8}$ UK Astronomy Technology Centre, Royal Observatory, Blackford Hill, Edinburgh EH9 3HJ, UK \\ ${ }^{9}$ Department of Astronomy, Cornell University, Ithaca, NY 14853, USA \\ Received 2013 July 3; accepted 2013 December 28; published 2014 January 27
}

\begin{abstract}
We mapped five massive star-forming regions with the SCUBA-2 camera on the James Clerk Maxwell Telescope. Temperature and column density maps are obtained from the SCUBA-2 450 and $850 \mu \mathrm{m}$ images. Most of the dense clumps we find have central temperatures below $20 \mathrm{~K}$, with some as cold as $8 \mathrm{~K}$, suggesting that they have no internal heating due to the presence of embedded protostars. This is surprising, because at the high densities inferred from these images and at these low temperatures such clumps should be unstable, collapsing to form stars and generating internal heating. The column densities at the clump centers exceed $10^{23} \mathrm{~cm}^{-2}$, and the derived peak visual extinction values are from 25 to $500 \mathrm{mag}$ for $\beta=1.5-2.5$, indicating highly opaque centers. The observed cloud gas masses range from $\sim 10$ to $10^{3} M_{\odot}$. The outer regions of the clumps follow an $r^{-2.36 \pm 0.35}$ density distribution, and this power-law structure is observed outside of typically $10^{4} \mathrm{AU}$. All these findings suggest that these clumps are high-mass starless clumps and most likely contain high-mass starless cores.
\end{abstract}

Key words: dust, extinction - H II regions - ISM: clouds - stars: formation - submillimeter: ISM

\section{INTRODUCTION}

The dust grains in clouds associated with massive starforming regions absorb the short-wavelength radiation from these stars, heat up, and then re-radiate at far-infrared (FIR) and sub-millimeter wavelengths. For this reason, clouds near H II regions are good sources of thermal emission. Since thermal radiation from dust is optically thin at FIR and sub-millimeter wavelengths, it is a good tracer of the temperature, density, and gas mass of the clouds, and these properties are significant in characterizing the early stages of star formation. Gas masses are usually determined from $\mathrm{CO}$ or dust continuum observations. However, $\mathrm{CO}$ is optically thick, its column density subject to an $X$-factor (Dickman 1978) that is not known (the Milky Way value is usually assumed) and is probably sensitive to the local metallicity or background UV field (van Dishoeck \& Black 1988; Leroy et al. 2009; Bolatto et al. 2013). Dust mass is usually derived from (sub)millimeter observations on the Rayleigh-Jeans side of the spectral energy distribution (SED), which are sensitive to dust temperatures down to 5-10 K, and in addition are probably also dependent on the metallicity.

Values of dust temperatures and masses estimated from dust emission are subject to the adopted values of the dust emissivity index, $\beta$. Values of $\beta$ in molecular clouds reflect different physical and chemical properties of the clouds, such as the grain size, structure, composition, and environment. At FIR wavelengths, the preferred value of $\beta$ in the diffuse interstellar medium is $\sim 2$ (Draine \& Lee 1984), and this is the commonly assumed value of $\beta$ at long wavelengths. However, a number of observations of cold cores (Shirley et al. 2005, 2011; Planck Collaboration et al. 2011) detected sub-millimeter dust emissivity indices greater than two. In cold, dense regions of molecular clouds, $\beta$ can be as steep as $\sim 3.7$ (Kuan et al. 1996) due to the accretion of ice mantles and grain growth. Toward circumstellar disks, the value of $\beta$ can be as flat as $\sim 1$ (Beckwith $\&$ Sargent 1991). Values of dust emissivity indices as low as $\sim 1$ are also observed toward Galactic cores with internal heating sources, perhaps caused by sightline variations in temperature (Juvela et al. 2011). Observations of some Class 0 young stellar objects (Kwon et al. 2009) and studies of circumstellar disks around Class II protostars, T Tauri stars (Ricci et al. 2010a, 2010b; Ubach et al. 2012), and young brown dwarfs (Ricci et al. 2012) in low-mass star-forming regions suggest even lower values of $\beta$, less than unity.

Although an accurate measurement of $\beta$ is critical in determining the physical properties of the clouds, the relationship between $\beta$ and dust temperature $\left(T_{\mathrm{d}}\right)$ is still under debate. Several studies suggest that $\beta$ and $T_{\mathrm{d}}$ are anti-correlated (Dupac et al. 2003; Désert \& et al. 2008; Paradis et al. 2010). However, such an inverse relation between $\beta$ and $T_{\mathrm{d}}$ resulting from leastsquares fits to flux densities is sensitive to noise and/or temperature variations along the line of sight (Shetty et al. 2009a, 2009b). A novel hierarchical Bayesian technique developed by Kelly et al. (2012) finds a weak positive correlation between $\beta$ and $T_{\mathrm{d}}$, but they use an unphysical assumption of isothermal graybody SED parameters. In their comprehensive study to examine the validity of five different potential methods on the shape of the $\beta-T_{\mathrm{d}}$ relation, Juvela et al. (2013) found an inverse $T_{\mathrm{d}}-\beta$ correlation with all the methods, and they noticed a lower bias especially when using the Bayesian method.

Numerous studies have shown that a substantial, but not very well constrained, fraction of the mass of the molecular material is in very cold clouds - at least as measured by the dust-and is not observable except at sub-millimeter wavelengths. 
One of the best-studied giant molecular cloud (GMC) H II regions is the Orion molecular cloud (OMC) - a moderately luminous region $\left(\sim 5 \times 10^{5} L_{\odot}\right.$ in the FIR), which is located at a distance of $420 \mathrm{pc}$. Using the Submillimeter High Angular Resolution Camera (SHARC), Lis et al. (1998) observed the Orion A molecular cloud, and their studies revealed an average dust temperature of $17 \pm 4 \mathrm{~K}$. Dust at temperatures down to $15 \mathrm{~K}$ is identified in a clump associated with Orion A (Mookerjea et al. 2000) from FIR observations at 138 and $205 \mu \mathrm{m}$ wavelengths. Mookerjea et al. also found signatures of emission at temperatures between 15 and $20 \mathrm{~K}$, corresponding to cold dust, in a number of Orion clumps, and virtually all of the dust mass is in this cold component. Orion is extensively analyzed using SCUBA by Johnstone et al. in a series of papers: the internal dust temperature of the clumps in the Orion B molecular cloud is in the range 20-40 K (Johnstone et al. 2001), whereas the best-fit temperature in the Orion B south molecular cloud is $18 \pm 4 \mathrm{~K}$ (Johnstone et al. 2006), and in Orion A clumps, this is $\sim 22 \pm 5$ (Johnstone \& Bally 2006). However, there is no overall census of the relative amounts of cold dust and warmer dust in clouds such as the OMC. One of the reasons for this is that it has been difficult to observe the extended emission, which is very extended in the case of the OMC.

Cold dust is observed in both low- and high-mass starforming regions, and the lowest temperatures found toward these regions differ only by a couple of kelvins. The dust temperatures measured toward low-mass star-forming regions are found to be as low as $\sim 7 \mathrm{~K}$ in a number of clouds (Evans et al. 2001), below $\sim 10-11 \mathrm{~K}$ in the pre-stellar cores of the $\varrho$ Oph main cloud (Stamatellos et al. 2007), and $~ 7 \mathrm{~K}$ in TMC-1C (Schnee et al. 2010). Over the past couple of years, a number of papers have focused on the physical properties of dust near low- and high-mass star-forming regions in the $250-500 \mu \mathrm{m}$ wavelength regime and have found dust temperatures as low as $10 \mathrm{~K}$ in molecular clouds. From Herschel Space Observatory (Pilbratt et al. 2010) data of a collection of low-mass cold, dense globules, Launhardt et al. (2013) obtained temperatures in the range $8-12 \mathrm{~K}$ at the interiors. The Balloon-borne Large Aperture Submillimeter Telescope (BLAST; Pascale et al. 2008) observations of an OB-forming complex, Cygnus-X, show some sources with temperatures as cold as $\sim 10 \mathrm{~K}$ (Roy et al. 2011). The "Herschel imaging survey of OB Young Stellar objects (HOBYS)" program (Motte et al. 2010) has provided unprecedented details of cold dust associated with $\mathrm{H}$ II regions. Using data from Herschel and other short- and long-wavelength data, the dust temperature map of the Galactic bubble H II region RCW 120, derived by Anderson et al. (2010), shows temperatures down to $\sim 10 \mathrm{~K}$ toward local infrared dark clouds (IRDCs). Vela-C, which is a site for both low- and high-mass star formation, shows warmer protostellar sources, with mean dust temperatures of $12.8 \mathrm{~K}$, compared to starless sources with mean dust temperatures of $10.3 \mathrm{~K}$ (Giannini et al. 2012). However, not all of these estimates of temperatures can be directly compared because of differences in wavelength coverage, modeling assumptions, and statistical treatments. In particular, the detection of very cold dust requires observations at longer wavelengths. Dust at temperatures below $10 \mathrm{~K}$ will have a peak in emission at wavelengths longer than $300 \mu \mathrm{m}$.

Observations of star formation in other galaxies are dominated by regions where clusters of massive stars heat their surrounding region. The properties measured for these regions, such as temperature, luminosity, mass, and star formation efficiency, are determined by the interactions of the massive stars and the molecular clouds near them. Understanding how to turn the observations into measured properties is highly dependent on what we have learned about star formation in similar massive star-forming regions in the Milky Way. However, only a handful of such regions have been well studied, and all of these studies suffer from a number of difficulties. To probe such regions, what we really require is higher spatial resolution and longer wavelength observations. In this paper, we use the new SCUBA-2 camera (Holland et al. 2013), at $450 \mu \mathrm{m}$ and $850 \mu \mathrm{m}$ wavelengths, to observe a carefully selected sample of massive star-forming regions in the outer Galaxy in order to overcome these problems.

In this paper, we calculate physical properties such as the temperature, density, and mass of the clouds associated with five massive star-forming regions in the outer Galaxy. The clouds are found within $15^{\prime}$ of $\mathrm{H}$ II regions, generally with no other significant concentration of interstellar material within many tens of arcminutes. We show the existence of very cold dust, with grain temperatures as low as $7.7 \mathrm{~K}$ near very hot massive star-forming regions.

\section{OBSERVATIONS AND DATA REDUCTION}

We used the SCUBA-2 instrument to observe five regions of massive star formation, centered on the H II regions: (1) S148, (2) S156, (3) S159, (4) S305, and (5) S254. Some of these regions only contain one $\mathrm{HII}$ region; some contain as many as five $\mathrm{H}_{\mathrm{II}}$ regions. These $\mathrm{H}$ II regions are located in the outer Galaxy, and there is very little confusion and contamination with foreground or background cold cores along the line of sight. The distances to our target objects are all well measured, and they are a few kiloparsecs (2.5-5.6) away from the Sun. The target objects are all very bright and could be easily mapped using SCUBA-2, since their angular sizes and separations between their component clumps are small. All of these objects have many observations at other wavelengths from optical to centimeter. The physical properties of all the $\mathrm{H}$ II regions are summarized in Table 1. Uncertainties on the linear diameter range from $\sim 5 \%$ to $27 \%$ with a mean uncertainty of $\sim 13 \%$ and are primarily due to the uncertainty in the distance to the $\mathrm{HII}$ region.

\subsection{SCUBA-2 Data}

We observed the area around S148, S156, S159, S305, and S254 as part of the SCUBA-2 guaranteed time project M11BGT01, primarily in weather bands 1 and 2, at various times between 2011 November 14 and 2012 March 14. The mean frequencies of the SCUBA-2 filters are $665 \mathrm{GHz}$ at $450 \mu \mathrm{m}$ waveband and $349.5 \mathrm{GHz}$ at $850 \mu \mathrm{m}$ waveband (see SCUBA-2 filter specifications). The half-power beam widths of the bandpass filters, corresponding to $\lambda / d \lambda \sim 14$ and 10 , are 32 and $85 \mu \mathrm{m}$, respectively, at 450 and $850 \mu \mathrm{m}$ (Holland et al. 2013). We used the rotating PONG (Kackley et al. 2010) method to map our target objects: the first three objects were observed in the PONG900 mode, which produced an image approximately $15^{\prime} \times 15^{\prime}$ in size, and in order to capture the extended features, the last two observations made use of the $30^{\prime} \times 30^{\prime}$ PONG1800 mapping mode. For some of the observations the beam size was observed to be larger than the nominal FWHM of 13.". $(850 \mu \mathrm{m})$ and 7".9 $(450 \mu \mathrm{m})$ (Dempsey et al. 2013): the James Clerk Maxwell Telescope (JCMT) beam sizes were $14^{\prime \prime} .2(850 \mu \mathrm{m})$ and 9..4 $(450 \mu \mathrm{m})$ for the first three observations, whereas the beam sizes were $14^{\prime \prime} .0(850 \mu \mathrm{m})$ and $7.5(450 \mu \mathrm{m})$ for the last two observations. 
Table 1

Properties of the Chosen H II Regions: H II Region Name, Distance, Angular Diameter, Physical Diameter, and Exciting Star

\begin{tabular}{|c|c|c|c|c|c|c|}
\hline Name & $\begin{array}{l}\text { R.A. } \\
\text { (J2000) }\end{array}$ & $\begin{array}{l}\text { Decl. } \\
\text { (J2000) }\end{array}$ & $\begin{array}{c}d \\
(\mathrm{kpc})\end{array}$ & $\begin{array}{l}\text { Diameter } \\
\left({ }^{\prime \prime}\right)\end{array}$ & $\begin{array}{l}\text { Diameter } \\
(\mathrm{pc})\end{array}$ & Exciting Star \\
\hline \multicolumn{7}{|c|}{ S148 complex } \\
\hline S147 & $22^{\mathrm{h}} 55^{\mathrm{m}} 40^{\mathrm{s}} \cdot 6$ & $58^{\circ} 28^{\prime} 02^{\prime \prime} 0$ & $5.50 \pm 0.89$ & 90 & 2.44 & $\mathrm{~A} 4 \mathrm{~V}^{\mathrm{a}}$ \\
\hline S148 & $22^{\mathrm{h}} 56^{\mathrm{m}} 05^{\mathrm{s}} .7$ & $58^{\circ} 30^{\prime} 55^{\prime \prime} .4$ & $5.60 \pm 0.3$ & 90 & 2.44 & $08 \mathrm{~V}^{\mathrm{a}}$ \\
\hline S149 & $22^{\mathrm{h}} 56^{\mathrm{m}} 22^{\mathrm{s}} \cdot 3$ & $58^{\circ} 31^{\prime} 57^{\prime \prime} .1$ & $5.60 \pm 0.3$ & 120 & 3.30 & $\mathrm{BO} \mathrm{V}^{\mathrm{a}}$ \\
\hline \multicolumn{7}{|c|}{ S156 complex } \\
\hline BFS15 & $23^{\mathrm{h}} 04^{\mathrm{m}} 42^{\mathrm{s}} \cdot 6$ & $60^{\circ} 04^{\prime} 56^{\prime \prime} .1$ & $3.0 \pm 0.7$ & 61 & 0.88 & \\
\hline S156 & $23^{\mathrm{h}} 05^{\mathrm{m}} 10^{\mathrm{s}} \cdot 6$ & $60^{\circ} 14^{\prime} 57^{\prime \prime} .6$ & $2.87 \pm 0.75$ & 40 & 0.56 & $\mathrm{O} 8 \mathrm{~V}^{\mathrm{b}}$ \\
\hline BFS18 & $23^{\mathrm{h}} 05^{\mathrm{m}} 47^{\mathrm{s}} .3$ & $60^{\circ} 24^{\prime} 06^{\prime \prime} .8$ & $3.0 \pm 0.7$ & 59 & 0.86 & $\ldots$ \\
\hline S159 & $23^{\mathrm{h}} 15^{\mathrm{m}} 31^{\mathrm{s}} .2$ & $61^{\circ} 06^{\prime} 55^{\prime \prime} .5$ & $2.97 \pm 0.31$ & 15 & 0.22 & O9 $\mathrm{V}^{\mathrm{b}}$ \\
\hline \multicolumn{7}{|c|}{ S254 complex } \\
\hline S254 & $06^{\mathrm{h}} 12^{\mathrm{m}} 20^{\mathrm{s}} \cdot 3$ & $18^{\circ} 02^{\prime} 32^{\prime \prime} .5$ & $2.46 \pm 0.16$ & 540 & 6.44 & $09.6 \mathrm{~V}^{\mathrm{c}}$ \\
\hline S255 & $06^{\mathrm{h}} 13^{\mathrm{m}} 09^{\mathrm{s}} .5$ & $17^{\circ} 58^{\prime} 40^{\prime \prime} 6$ & $2.46 \pm 0.16$ & 300 & 3.58 & $\mathrm{~B} 0.0 \mathrm{~V}^{\mathrm{c}}$ \\
\hline S256 & $06^{\mathrm{h}} 12^{\mathrm{m}} 39^{\mathrm{s}} \cdot 3$ & $17^{\circ} 56^{\prime} 48^{\prime \prime} .1$ & $2.46 \pm 0.16$ & 120 & 1.43 & $\mathrm{~B} 0.9 \mathrm{~V}^{\mathrm{c}}$ \\
\hline S257 & $06^{\mathrm{h}} 12^{\mathrm{m}} 49^{\mathrm{s}} .1$ & $17^{\circ} 58^{\prime} 46^{\prime \prime} .1$ & $2.46 \pm 0.16$ & 240 & 2.86 & $\mathrm{~B} 0 \mathrm{~V}^{\mathrm{d}, \mathrm{e}}$ \\
\hline S258 & $06^{\mathrm{h}} 13^{\mathrm{m}} 33^{\mathrm{s}} \cdot 6$ & $17^{\circ} 55^{\prime} 39^{\prime \prime} .7$ & $2.46 \pm 0.16$ & 59 & 0.7 & $\mathrm{~B} 1.5 \mathrm{~V}^{\mathrm{c}}$ \\
\hline S305 & $07^{\mathrm{h}} 30^{\mathrm{m}} 07^{\mathrm{s}} .0$ & $-18^{\circ} 31^{\prime} 33^{\prime \prime} .5$ & $5.20 \pm 1.40$ & 420 & 10.6 & $08.5 \mathrm{~V}, 09.5 \mathrm{~V}$ \\
\hline
\end{tabular}

Notes. The exciting star of S147 is an A4 V star; however, this exciting star cannot produce a H II region and therefore S147 is most likely ionized by a star of a different spectral type.

${ }^{\text {a }}$ Crampton et al. (1978).

${ }^{\mathrm{b}}$ Russeil et al. (2007).

${ }^{\text {c }}$ Chavarría et al. (2008).

${ }^{\mathrm{d}}$ Pismis \& Hasse (1976).

${ }^{\mathrm{e}}$ Moffat et al. (1979).

${ }^{\mathrm{f}}$ Russeil et al. (1995).

The SCUBA-2 data were reduced using the Starlink SMURF (version 1.3.8 for the first three observations and version 1.3.10 for the last two observations) and the iterative map maker (Chapin et al. 2013), which is part of the sub-millimeter user reduction facility SMURF (Jenness et al. 2011) software package. We followed the recommended "best practice" (Thomas 2013) and used an externally generated zero mask to constrain the map maker and prevent negative bowling.

The final maps were calibrated using flux calibration factors (FCFs) obtained from planet data for the respective observation dates. We derived the FCF using SMURF and the pipeline for combining and analyzing reduced data, PICARD (Jenness et al. 2008), ${ }^{10}$ which is part of the ORAC-DR (Jenness \& Economou 1999) pipeline and has the same infrastructure. The FCFs for S156 were derived at both wavelengths using the primary calibrator Mars on the night of the science observation; Uranus was also available as a primary calibrator. Due to bad focusing and atmospheric effects in the Uranus observation (seen primarily at $450 \mu \mathrm{m}$ ), which was taken before the period of the science observation in the early evening hours of the observation date, we selected a midnight Mars observation to derive the FCF. In addition, there were standard peak FCF values available from nearly 500 observations at both wavelengths (Dempsey et al. 2013). The standard FCFs corresponding to the Starlink SMURF version at the time of data reduction were $(556 \pm 45) \mathrm{Jy}_{\text {beam }}{ }^{-1} \mathrm{pW}^{-1}$ and $(606 \pm 55) \mathrm{Jy}_{\text {beam }}{ }^{-1}$ $\mathrm{pW}^{-1}$ at 850 and $450 \mu \mathrm{m}$, respectively. The values of the FCFs used for calibrating S156 are $545 \mathrm{Jy}_{\text {beam }}{ }^{-1} \mathrm{pW}^{-1}$ at $850 \mu \mathrm{m}$ and $563 \mathrm{Jy}$ beam $^{-1} \mathrm{pW}^{-1}$ at $450 \mu \mathrm{m}$, and these are within the uncertainty limits of the standard values. The FCFs for S159 and S148, which were observed on the same day, obtained from both

\footnotetext{
10 http://www.oracdr.org/oracdr/PICARD
}

Mars and Uranus, displayed large variations from the standard value, especially at $450 \mu \mathrm{m}$. We therefore adopted the standard FCFs to calibrate S159 and S148 data.

In finding the FCFs of S305 and S254, we considered two different observations of the planet Mars acquired on the same night. We adopted the FCF derived from the early night Mars observation for calibrating the short-wavelength data. For calibrating the long-wavelength data, we chose a Mars observation near midnight. The values of the FCFs used to calibrate S305 and S254 data at 850 and $450 \mu \mathrm{m}$ wavelengths are, respectively, 566 and $565 \mathrm{Jy}_{\text {beam }}{ }^{-1} \mathrm{pW}^{-1}$. The effect of FCF uncertainty on the final fluxes is less than $5 \%$ at $850 \mu \mathrm{m}$ and $10 \%$ at $450 \mu \mathrm{m}$ (Dempsey et al. 2013).

\subsection{Role of Background on SCUBA-2 Data}

We chose data from one of the objects observed, S305 (the object with the most widespread emission structures), to examine in detail the effects of background emission on the measurements made from the images. We measured the flux density in same-sized apertures at numerous positions showing no discreet emission that might be due to real objects. When we increase the distance from the center of the image, the mean background flux densities approach positive values. At the edges of the maps, especially near $25^{\prime}$, the background emission is the highest and is most likely due to the reduced integration time. The error retains a constant value up to around $20^{\prime}$ from the map center and then begins to increase out to the edge. The mean background values at $450 \mu \mathrm{m}$ show much more variability than the corresponding values at $850 \mu \mathrm{m}$. The background standard deviation, however, maintains a constant value up to around $20^{\prime}$ from the map center and then increases up to the map boundary. The mean background value of the S305 
field $\left(\sim-0.003 \mathrm{Jy}\right.$ beam $^{-1}$ at $850 \mu \mathrm{m}$ and $\sim-0.009 \mathrm{Jy} \mathrm{beam}^{-1}$ at $450 \mu \mathrm{m})$ is not significantly different from zero, relative to

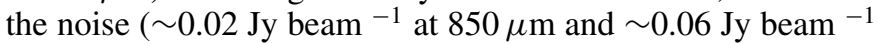
at $450 \mu \mathrm{m})$. Thus, on average, the background emission values are close to zero; therefore, we did not take into account the contribution due to background in the $\mathrm{S} 305$ source emission.

In all the target fields of our study, the contribution from the mean value of the background on the source flux is computed to be very low and is neglected in further calculations. Typical on-source signal, especially in the fainter extended regions of $\mathrm{S} 305$, is $(0.12 \pm 0.04) \mathrm{Jy}$ beam $^{-1}$ at $850 \mu \mathrm{m}$ and $(0.36 \pm 0.10) \mathrm{Jy}$ beam $^{-1}$ at $450 \mu \mathrm{m}$. Note that the absolute value of the mean background emission in S305 is a factor of around 40 (at 850 and $450 \mu \mathrm{m}$ ) times smaller than the faint extended on-source emission. For all the target objects, the ratio of the faintest on-source signal to the mean background, on average, is around 41 at $850 \mu \mathrm{m}$ and 17 at $450 \mu \mathrm{m}$. The total flux due to this offset in the background may be significant for a measure of the integrated flux from these objects, but our main result, the low temperatures of the centers of the clouds, depends on the ratios of surface brightness.

\section{PROPERTIES OF THE DUST DERIVED FROM THE IMAGES}

Since dust emission at sub-millimeter wavelengths is optically thin, it is a good tracer of the masses of the clouds. In order to estimate temperature, density, and mass, we employed the surface brightness maps of the clouds obtained from SCUBA-2 at $450 \mu \mathrm{m}$ and $850 \mu \mathrm{m}$ wavelengths. In this section we describe the clouds' physical properties derived using the observed sub-millimeter dust emission maps. The properties are dependent on environmental and instrumental factors, such as calibration, line contamination, knowledge of $\beta$, effect of foreground/background, and temperature variations along the line of sight, all of which are examined at the end of Section 3.3 and discussed in Section 4.

\subsection{Dust Emission Morphology}

The five observed SCUBA-2 maps at 450 and $850 \mu \mathrm{m}$ are displayed in Figures 1(a)-5(a) and 2(b)-5(b). Hereafter, north is up and east is to the left, with map coordinates in J2000. Due to large-scale sky variations in the $450 \mu \mathrm{m}$ map of S148, we discarded this map from further analysis. A long bar-like cloud whose strongest emission is located at $22^{\mathrm{h}} 56^{\mathrm{m}} 45^{\mathrm{s}} .8$ R.A. and $+58^{\circ} 30^{\prime}$ decl., aligned in the north-south direction, is visible in the southeast direction of the cloud associated with the H II region S148. A third cloud is present in the S148 field at a distance of $\sim 6^{\prime} 45^{\prime \prime}$ from the map center to the north. Please note that there are no visible optical counterparts for these two unknown clouds in the DSS red image, and therefore these two clouds are most likely some dark clouds along the line of sight or associated with the center cloud. Since the identity and the distances to these clouds are uncertain, we are not taking these two clouds into account in further studies. In the $\mathrm{HI}$ region clouds under discussion, it is apparent that the $450 \mu \mathrm{m}$ maps resemble their $850 \mu \mathrm{m}$ counterparts. The maps presented here are in units of $\mathrm{Jy}_{\text {beam }}{ }^{-1}$, and to convert these values to units of $\mathrm{MJy} \mathrm{sr}^{-1}$ (the other commonly used units), one has to multiply by 185 at $850 \mu \mathrm{m}$ and 426 at $450 \mu \mathrm{m}$ for S159, S148, and S156. For S305 and S254, the Jy beam ${ }^{-1}$ values need to be multiplied by 192 at $850 \mu \mathrm{m}$ and 668 at $450 \mu \mathrm{m}$ to obtain corresponding values in $\mathrm{MJy} \mathrm{sr}^{-1}$.
Table 2

Observed Parameters ${ }^{\mathrm{a}}$

\begin{tabular}{lccccc}
\hline \hline Source & $\begin{array}{c}\text { R.A. } \\
(\mathrm{J} 2000)\end{array}$ & $\begin{array}{c}\text { Decl. } \\
(\mathrm{J} 2000)\end{array}$ & $\begin{array}{c}S_{850}^{\text {peak }} \\
\left(\mathrm{Jy} \mathrm{beam}^{-1}\right)\end{array}$ & $\begin{array}{c}S_{450}^{\text {peak }} \\
\left(\mathrm{Jy} \mathrm{beam}^{-1}\right)\end{array}$ & $\begin{array}{c}T_{\mathrm{cd}} \\
(\mathrm{K})\end{array}$ \\
\hline S148 & $22^{\mathrm{h}} 56^{\mathrm{m}} 17^{\mathrm{s}} .2$ & $58^{\circ} 31^{\prime} 01^{\prime \prime} .0$ & 0.50 & $\ldots$ & $\ldots$ \\
S156 & $23^{\mathrm{h}} 05^{\mathrm{m}} 08^{\mathrm{s}} .9$ & $60^{\circ} 14^{\prime} 48^{\prime \prime} .9$ & 1.09 & 3.49 & 11.5 \\
BFS15 & $23^{\mathrm{h}} 05^{\mathrm{m}} 24.6$ & $60^{\circ} 08^{\prime} 12^{\prime \prime} .8$ & 2.09 & 5.48 & 9.5 \\
S156NE & $23^{\mathrm{h}} 06^{\mathrm{m}} 18^{\mathrm{s}} .7$ & $60^{\circ} 16^{\prime} 17^{\prime \prime} .8$ & 0.35 & $\ldots$ & $\ldots$ \\
S159 & $23^{\mathrm{h}} 15^{\mathrm{m}} 30.7$ & $61^{\circ} 07^{\prime} 14^{\prime \prime} .8$ & 2.34 & 9.03 & 14.3 \\
S254N & $06^{\mathrm{h}} 12^{\mathrm{m}} 53.6$ & $18^{\circ} 00^{\prime} 27^{\prime \prime} .7$ & 4.97 & 21.7 & 24.6 \\
S254S1 & $06^{\mathrm{h}} 12^{\mathrm{m}} 53.8$ & $17^{\circ} 59^{\prime} 24^{\prime \prime} .7$ & 5.33 & 23.4 & 23.4 \\
S254S2 & $06^{\mathrm{h}} 12^{\mathrm{m}} 56^{\mathrm{s}} .8$ & $17^{\circ} 58^{\prime} 34^{\prime \prime} .0$ & 1.29 & 4.94 & 28.6 \\
S305N & $07^{\mathrm{h}} 29^{\mathrm{m}} 56^{\mathrm{s}} .2$ & $-18^{\circ} 27^{\prime} 54^{\prime \prime} .2$ & 0.71 & 2.22 & 13.7 \\
S305W1 & $07^{\mathrm{h}} 30^{\mathrm{m}} 00^{\mathrm{s}} .3$ & $-18^{\circ} 33^{\prime} 33^{\prime \prime} 5$ & 0.33 & 0.84 & 13.8 \\
S305W2 & $07^{\mathrm{h}} 30^{\mathrm{m}} 00.4$ & $-18^{\circ} 31^{\prime} 09^{\prime \prime} .5$ & 0.26 & 0.67 & 14.7 \\
S305W3 & $07^{\mathrm{h}} 30^{\mathrm{m}} 00.6$ & $-18^{\circ} 31^{\prime} 54^{\prime \prime} .5$ & 0.37 & 1.01 & 14.1 \\
S305W4 & $07^{\mathrm{h}} 30^{\mathrm{m}} 00^{\mathrm{s}} .9$ & $-18^{\circ} 34^{\prime} 44^{\prime \prime} .5$ & 0.18 & 0.53 & 12.9 \\
S305W5 & $07^{\mathrm{h}} 30^{\mathrm{m}} 04^{\mathrm{s}} .8$ & $-18^{\circ} 31^{\prime} 03^{\prime \prime} .5$ & 0.29 & 0.84 & 13.8 \\
S305E1 & $07^{\mathrm{h}} 30^{\mathrm{m}} 11^{\mathrm{s}} .8$ & $-18^{\circ} 32^{\prime} 36^{\prime \prime} 3$ & 0.38 & 1.04 & 14.3 \\
S305S & $07^{\mathrm{h}} 30^{\mathrm{m}} 16^{\mathrm{s}} .5$ & $-18^{\circ} 35^{\prime} 51^{\prime \prime} .2$ & 1.10 & 3.82 & 16.7 \\
S305E2 & $07^{\mathrm{h}} 30^{\mathrm{m}} 16.7$ & $-18^{\circ} 33^{\prime} 54^{\prime \prime} .3$ & 0.13 & 0.37 & 14.5 \\
\hline
\end{tabular}

Notes. ${ }^{\text {a }}$ Clouds associated with H II regions; peak positions; 850 and $450 \mu \mathrm{m}$ peak flux densities of the clouds detected in the SCUBA-2 observations of Galactic $H_{\text {II }}$ region complexes; and dust temperatures, $T_{\mathrm{cd}}$ (for $\beta=2$ ), which come from the peak 450 and $850 \mu \mathrm{m}$ emission. See Figures $1-5$ for maps of these fields.

The observed characteristics of the clouds in the vicinity of the individual $\mathrm{H}$ II regions are thoroughly discussed in Sreenilayam (2012) and are tabulated in Table 2, where the peak positions and flux densities for each object are given. The uncertainties in the peak flux densities are primarily due to uncertainties in the FCF: i.e., $\sim 5 \%$ at $850 \mu \mathrm{m}$ and $10 \%$ at $450 \mu \mathrm{m}$ (Dempsey et al. 2013). Note that many of these images show more than one area of significant emission. Some of these are more closely associated with other nearby $\mathrm{H}$ II regions, and we have indicated these with the appropriate names in this table. Some of these emission features are roughly circular and isolated, and later in this paper we identify them as "clumps" and analyze their properties. We presume that these clumps are gravitationally bound collections of cores, and interferometric observations may reveal their fragmentation into individual cores at arcsec resolution. Throughout the paper "cloud" is a general term that, in addition to clumps, refers to less symmetric objects (although some of those might be more correctly described as filaments).

\subsubsection{Temperature Distribution}

One can derive a pixel-to-pixel dust color temperature map of the clouds by taking the ratio of $450-850 \mu \mathrm{m}$ observed surface brightness maps:

$$
\frac{S_{450}(\mathrm{Jy} / 450 \text { beam })}{S_{850}(\mathrm{Jy} / 850 \text { beam })}=\left(\frac{850}{450}\right)^{3+\beta} \frac{\exp \left(17 K / T_{\mathrm{d}}\right)-1}{\exp \left(32 K / T_{\mathrm{d}}\right)-1}
$$

(Kramer et al. 2003).

Before constructing the ratio maps, we first smoothed the $450 \mu \mathrm{m}$ SCUBA-2 map to the $850 \mu \mathrm{m}$ resolution of the JCMT beam using a symmetrical Gaussian point-spread function to roughly match the Gaussian main beams at both wavelengths. The error beams, however, are not taken into account in matching the fluxes at two wavelengths. The flux ratio is modeled assuming that the measured flux density at both wavelengths is due to thermal emission from optically thin 

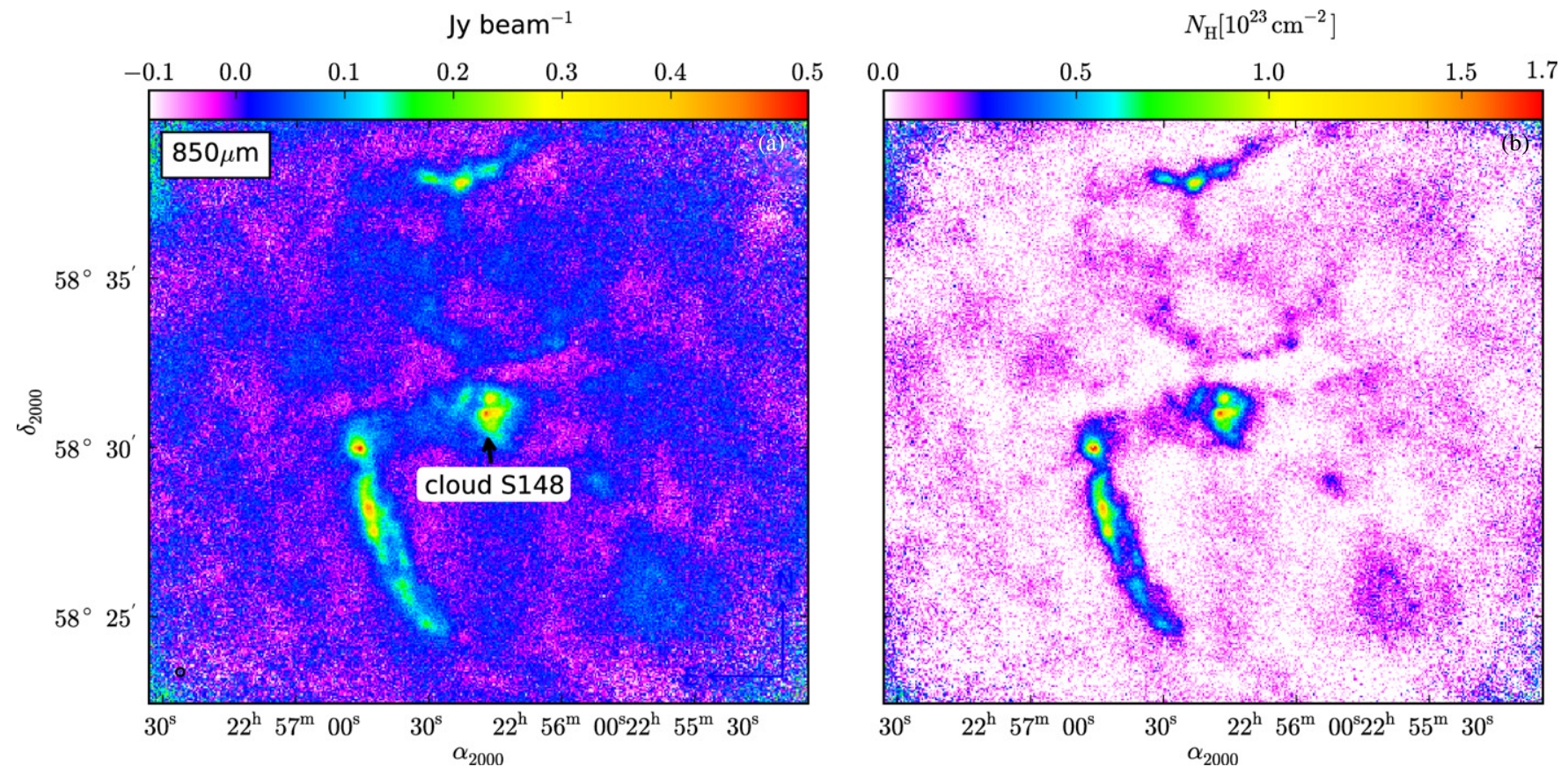

Figure 1. S148: (a) surface brightness map at $850 \mu \mathrm{m}$ and (b) column density map of S148 at $850 \mu \mathrm{m}$ using temperature derived from assuming a $T_{\mathrm{d}}$ of $10 \mathrm{~K}$.

dust grains and is dependent on the dust emissivity index and temperature. In all the temperature maps, the distribution is derived by assuming a constant value for the dust emissivity index $\beta$. Note that the cloud S148 lacks a good detection at $450 \mu \mathrm{m}$, and so we were unable to provide a detailed dust temperature map for this cloud. To derive the dust physical properties, we considered a $\beta$ value of 2 , which is often considered to be the standard value at sub-millimeter and millimeter wavelengths (Draine \& Lee 1984). The effect of varying emissivity index is discussed in Section 3.3 in order to investigate the effect of the assumed value of $\beta$ on the calculated values of the temperature, density, and mass. The variations in the dust temperature with the flux ratio values are dependent on the adopted values of $\beta$. If the $450 / 850$ flux ratio is above $\approx 8.3$, $11.5,15.6$ for $\beta=1.5,2,2.5$, respectively, then no temperature can be found with the assumptions used here (e.g., uniform dust and dust opacity along the line of sight).

Dust color $(450 / 850)$ temperature maps of the clouds are calculated using a single dust emissivity index, $\beta=2.0$, and assuming that SCUBA-2 emission at 450 and $850 \mu \mathrm{m}$ are solely originating from dust grains at a single effective temperature. The derived temperatures represent the average of the observed temperatures along the line of sight through the cloud depths (see Figures 2(c), 3(c), 4(c), and 5(c)) but with some additional weighting, of an unknown amount, on the hotter outer parts of each cloud.

There are just a few, almost random, points at the outer edges of each structure, where the signal-to-noise ratio $(\mathrm{S} / \mathrm{N})$ is low, that are well over $100 \mathrm{~K}$, and they may be just the result of noise. To improve our display of the interior regions, we have set the maximum in the color tables for these figures at $80 \mathrm{~K}$, except for S254, where we have set the maximum to $150 \mathrm{~K}$ since the temperatures are much higher in the central regions of this complex. For most of the structures shown, this has resulted in 2 or 3 pixels "disappearing" at the noisy edges, but in the case of S159, approximately 20 "hot" pixels in the outer edge are not shown. In each cloud, a gradient in temperature is observed increasing from the cloud center to the periphery.
Temperatures are not derived for the cloud associated with S148, due to large-scale sky variations in the $450 \mu \mathrm{m}$ map. We assume a uniform temperature of $10 \mathrm{~K}$ throughout S148 to derive the rest of the dust characteristics at sub-millimeter wavelengths. Most of the sources in the S156 field show dust temperature gradients (see Figure 2(c)). The clump near the $\mathrm{H}$ II region $\mathrm{S} 156$ is cold, with central temperatures $\sim 11.5 \mathrm{~K}$, and most of the interior of this clump is below $20 \mathrm{~K}$. The extended region of the clump S156, located in the southeast direction, is relatively warm-reaching on average $\sim 43 \mathrm{~K}$ with some pixels at the edge showing temperatures of $\sim 79 \mathrm{~K}$. Note that the clump associated with the H II region BFS15, located in the south, is showing a central temperature of $9.5 \mathrm{~K}$ and $\sim 28 \mathrm{~K}$, on average, at the outer layers. The $450 \mu \mathrm{m}$ emission of the cloud S156NE is weak $\left(\sim 1 \mathrm{Jy}^{\text {beam }}{ }^{-1}\right)$ and uniform, while the $850 \mu \mathrm{m}$ emission, although weak, does show a peak in emission. There are a couple of pixels near the center of S156NE showing temperatures below $30 \mathrm{~K}$, possibly due to noise in this generally low $\mathrm{S} / \mathrm{N}$ region; most of the interior of S156NE shows temperatures of 30-60 K with an increase toward the outer edges. Temperature variations are high in the clump associated with S159, with some of the pixels at the main clump's outer boundary reaching around $80 \mathrm{~K}$. However, the pixel at the center with the lowest value of $T_{\mathrm{d}}$ is at $14.3 \mathrm{~K}$. Outer parts of the clump retain a temperature between 30 and $60 \mathrm{~K}$. All the fragments located in the southwest and northeast directions of the primary cloud show lowest temperatures of around $10 \mathrm{~K}$. The minor fragments visible in the southeast direction are significantly warmer: the temperatures of these regions are found to be above $20 \mathrm{~K}$ (see Figure 3(c)). S159 is selected for more detailed analysis in Sections 3.2 and 3.3, as this is the largest, and therefore best-resolved, bright, circularly symmetric object.

The centers of the clumps associated with the S254 complex, primarily S254N, S254S1, and S254S2, are warmer than the centers of clumps associated with other $\mathrm{H}$ II regions in this study. The centers are at temperatures $\sim 23 \mathrm{~K}$ (S254S1), $25 \mathrm{~K}$ (S254N), and $29 \mathrm{~K}(\mathrm{~S} 254 \mathrm{~S} 2)$, and a couple of pixels at the exterior 

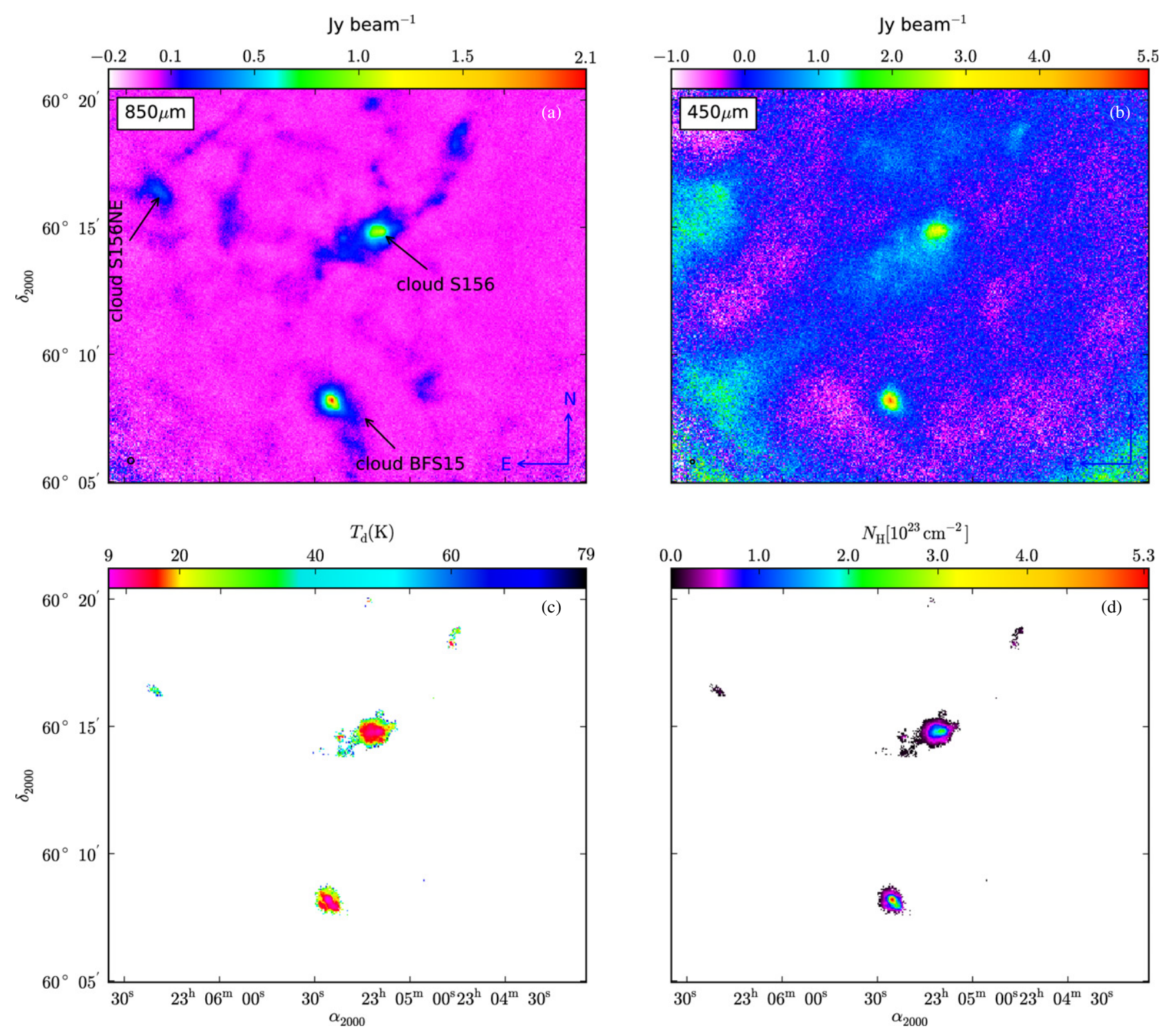

Figure 2. S156: (a) surface brightness map at $850 \mu \mathrm{m}$, (b) surface brightness map at $450 \mu \mathrm{m}$, (c) dust temperature map, and (d) column density map.

boundary show temperatures beyond $100 \mathrm{~K}$ (Figure 4(c)). These warmer clumps are most likely associated with the presence of two hot $\mathrm{HI}$ regions on either side of the clouds. This is the hottest environment in our sample, as these clumps are surrounded not only by these two much larger HiI regions, but also by several other large $\mathrm{H}$ II regions in the immediate vicinity. The cloud fragment in the southeast direction, at R.A. $06^{\mathrm{h}} 13^{\mathrm{m}} 29^{\mathrm{s}} .2$ and decl. $+17^{\circ} 55^{\prime} 30^{\prime} .8$, connected to the H II region S258, shows similar characteristics, with around $23 \mathrm{~K}$ dust at its center. However, the cloud located in the southeast direction at R.A. $06^{\mathrm{h}} 13^{\mathrm{m}} 47^{\mathrm{s}} .0$ and decl. $+17^{\circ} 54^{\prime} 54^{\prime \prime} .5$ shows a relatively colder central temperature of $\sim 11 \mathrm{~K}$. The minimum values of dust temperatures in the clouds of the S305 complex range from $\sim 12 \mathrm{~K}$ to $17 \mathrm{~K}$. The clump at the southeast (S305S) is the warmest among all other nebulosities, with central temperatures of $\sim 17 \mathrm{~K}$ and grain temperatures reaching $\sim 46 \mathrm{~K}$ (on average) at the boundary. All other components of the complex are colder than S305S, with central temperatures ranging from $\sim 12$ to $14 \mathrm{~K}$ (Figure 5(c)).

We identify 15 clouds with good $\mathrm{S} / \mathrm{N}$ in both 450 and $850 \mu \mathrm{m}$ wavelengths (listed in Table 2). We find that 11 of these have central temperatures, $T_{\mathrm{cd}}$, below $15 \mathrm{~K}$, and only 3 have $T_{\mathrm{cd}}$ above $20 \mathrm{~K}$ (typical uncertainties in $T_{\mathrm{cd}}$ are about $11 \%$ ). Temperatures below $20 \mathrm{~K}$ strongly suggest that there is no central heating source such as an embedded protostar. The average value of the dust temperatures at the centers of all the clouds associated with $\mathrm{H}$ II regions is $\sim 16 \mathrm{~K}$. The lowest dust temperature is at the center of the clump BFS15 $(9.5 \mathrm{~K})$, and the warmest one is in the S254 complex, S254S2 $(28.6 \mathrm{~K})$. In all the objects the pixel with the lowest temperature can be driven by noise, and to improve the estimate of the clump, we average over a 14 " diameter region; this typically produced a temperature $\sim 0.7-3.6 \mathrm{~K}$ higher. However, in the central clumps of the S254 complex, this produced $\sim 7-25 \mathrm{~K}$ higher temperature.

Line-of-sight averaged dust temperature $\left(\left\langle T_{\mathrm{d}}\right\rangle_{\text {los }}\right)$ profiles of all the clumps are derived by assuming that each clump has a spherical structure. The profile values are obtained by averaging the line-of-sight observed temperatures over concentric annuli of width 0.5 times the $850 \mu \mathrm{m}$ FWHM centered on the clump, and with uncertainties in the temperature values representing the quadratic sum of the errors due to calibration and the standard deviation from the cylindrical symmetry at each radial annulus. 

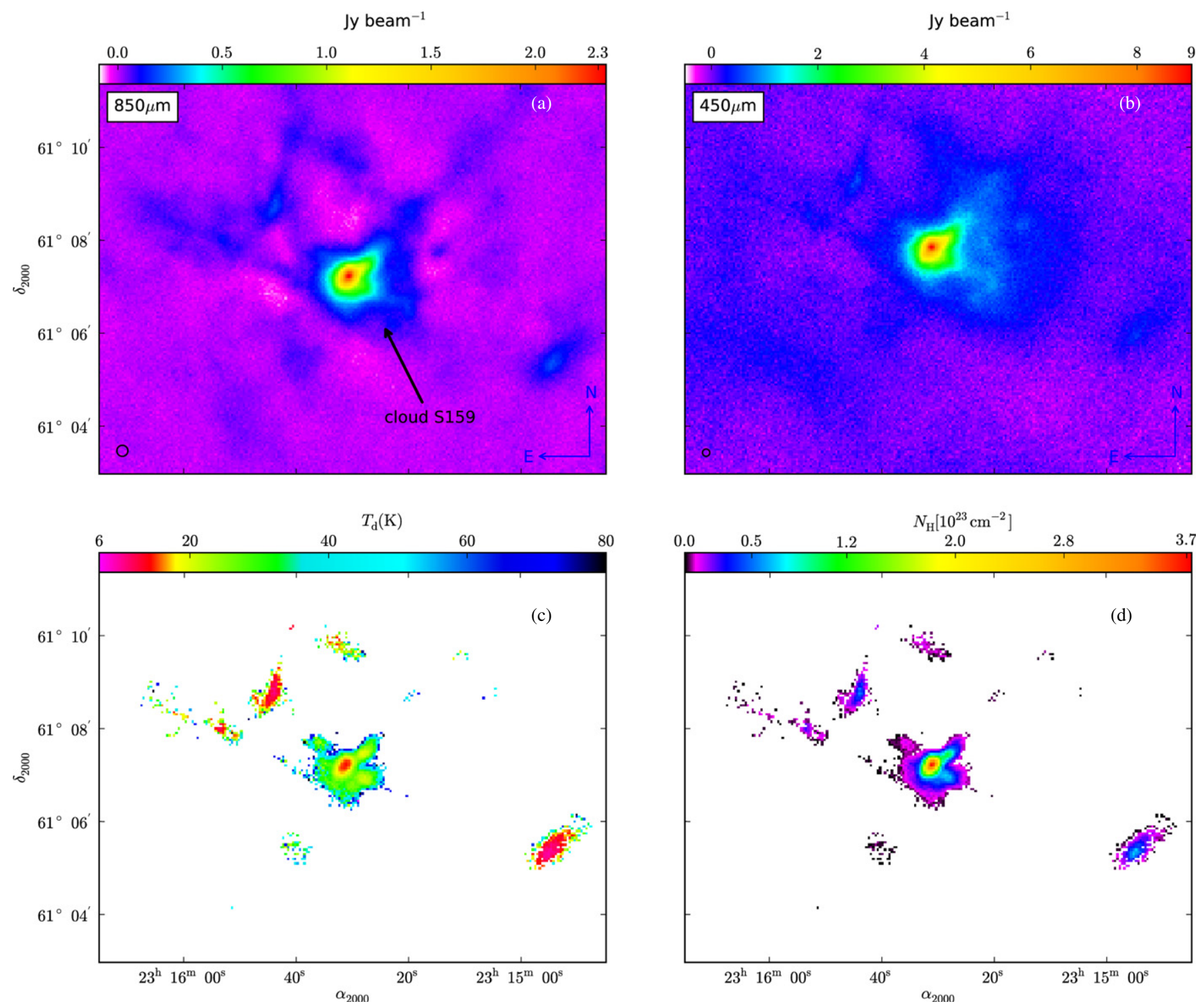

Figure 3. S159: (a) surface brightness map at $850 \mu \mathrm{m}$, (b) surface brightness map at $450 \mu \mathrm{m}$, (c) dust temperature map, and (d) column density map.

The majority of the clumps have $\left\langle T_{\mathrm{d}}\right\rangle_{\text {los }}$ less than $\sim 28 \mathrm{~K}$, with temperatures ranging from $\sim 13-28 \mathrm{~K}$ (in the center) to $\sim 25-50 \mathrm{~K}$ (at the edges) except the clumps in the central regions of the $\mathrm{S} 254$ complex, where the $\left\langle T_{\mathrm{d}}\right\rangle_{\text {los }}$ are higher, with $\sim 42-54 \mathrm{~K}$ at the center and $\sim 90-100 \mathrm{~K}$ at the clump boundary.

The observed $\left\langle T_{\mathrm{d}}\right\rangle_{\text {los }}$ profile of one of the clumps (near S159) for $\beta=2$ is shown in Figure 7(a) (red curve), where we examine the effect of line contamination and $\beta$ on our results (as discussed below). The observed $\left\langle T_{\mathrm{d}}\right\rangle_{\text {los }}$ distribution shows a rise in the temperature from the clump's center to the outer envelope, indicating less input heating in the center, for a constant value of the emissivity index. This feature in our target clumps is most likely attributed to the lack of any central heating source, since cores with increasing temperatures toward smaller radii represent cores that enclose central heating sources (e.g., van der Tak et al. 2000). However, in our clumps, there may be external radiation fields, due to the exciting stars of the nearby H II regions. The only clouds in our sample that would have higher central temperatures due to this are those with lower columns and/or higher external radiation fields, and both of these conditions appear to be true for the sources in the central filament of S254. The range of temperatures found in our study is slightly higher than that obtained from the low-mass starless core TMC-1C in the Taurus molecular cloud: for a $\beta$ of 1.5 , they observe dust temperatures ranging from $6 \mathrm{~K}$ to $15 \mathrm{~K}$ from the center to the outer envelope (Schnee \& Goodman 2005), whereas our study on $\mathrm{S} 159$ for a $\beta$ of 2 reveals somewhat higher dust temperatures, ranging from $\sim 14 \mathrm{~K}$ to $50 \mathrm{~K}$ from the center to the outer envelope.

\subsubsection{Column Density Distribution}

We calculated the cloud optical depth assuming that the dust emission is optically thin at SCUBA-2 wavelengths. The $850 \mu \mathrm{m}$ map (which is in units of $\mathrm{Jy} \mathrm{beam}^{-1}$ ) is selected to calculate the beam-averaged optical depth. Using the $850 \mu \mathrm{m}$ flux density, the beam area at $850 \mu \mathrm{m}$, the Planck function at $850 \mu \mathrm{m}\left(B_{850}\right)$, and the $450 / 850$ color temperature found above, we can derive the optical depth at $850 \mu \mathrm{m}\left(\tau_{850}\right)$ :

$$
\tau_{850}=\frac{F_{850}}{\Omega_{\mathrm{b}} B_{850}\left(T_{\mathrm{d}}\right)} .
$$

Since thermal emission from dust at such long wavelengths is optically thin, $\tau_{850} \ll 1$, sub-millimeter dust emission 

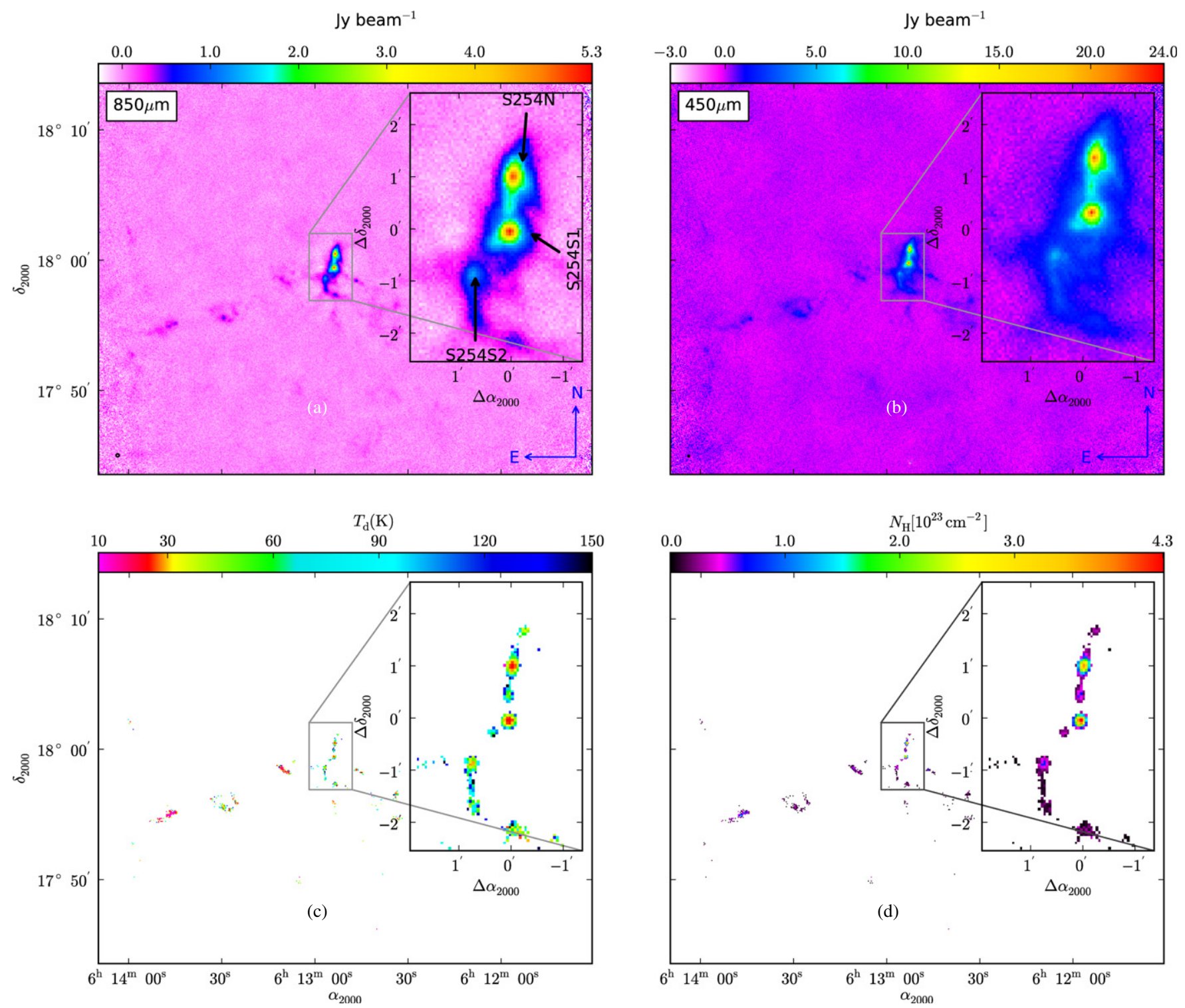

Figure 4. S254: (a) surface brightness map at $850 \mu \mathrm{m}$, (b) surface brightness map at $450 \mu \mathrm{m}$, (c) dust temperature map, and (d) column density map. The center for the inset map is at R.A. $06^{\mathrm{h}} 12^{\mathrm{m}} 53^{\mathrm{s}} .9$ and decl. $17^{\circ} 59^{\prime} 24^{\prime \prime} .7$.

can follow high column densities, and the column density of hydrogen $(\mathrm{H})$ from $850 \mu \mathrm{m}$ observations can be derived directly from the optical depth using the formula

$$
N_{\mathrm{H} 850}=\frac{\tau_{850}}{\kappa_{850} \mu m_{\mathrm{H}}},
$$

where $\kappa_{850}$ is the mass absorption coefficient, the opacity per unit mass column density, at $850 \mu \mathrm{m}$. We adopt $\kappa_{850}=0.01 \mathrm{~cm}^{2} \mathrm{~g}^{-1}$ for a gas-to-dust mass ratio of 100 (opacity per unit dust mass, $\left.\kappa_{850, \mathrm{~d}}=1 \mathrm{~cm}^{2} \mathrm{~g}^{-1}\right)$ in the column density calculations of the target objects. The quantity $\mu$ (2.33) is the mean molecular weight of the cloud material, and $m_{\mathrm{H}}$ is the mass of a hydrogen atom. The measured column density is proportional to the flux at a particular wavelength, as well as the temperature of the dust. We also estimate the extinction $A_{V}$ at each pixel position using the widely accepted Milky Way value of $R_{V}=3.1$ in calculations; any change in $R_{V}$, therefore, changes the value of visual extinction, $A_{V}$. For example, if we adopt an $R_{V}$ of 5.5, as found for denser star-forming regions (Mathis 1990;
Draine 2003; Whittet 2003), in our calculations, then the visual extinction of the clouds will be modified by a factor of $\sim 2$.

The maps of the hydrogen column density $N_{\mathrm{H}}$ derived from the $850 \mu \mathrm{m}$ optical depth maps are shown in Figures 1(d), 2(d), 3(d), 4(d), and 5(d). The value of $N_{\mathrm{H}}$ at the outer cloud boundary is very low, $\approx 10^{21} \mathrm{~cm}^{-2}(\mathrm{~S} 148, \mathrm{~S} 156, \mathrm{~S} 159, \mathrm{~S} 305)$ and $\approx 10^{22} \mathrm{~cm}^{-2}$ (central regions of the $\mathrm{S} 254$ complex). The column densities approach their highest values toward the centers of the clumps. Note that a column density of $\approx 10^{21} \mathrm{~cm}^{-2}$ corresponds to an extinction of less than one magnitude, and thus these observed edges of the clouds should match very well with the cloud edges that one would find in an optical image. In fact, in the 450 and especially in the $850 \mu \mathrm{m}$ images we do see slightly farther out than we can accurately compute the temperature and column density, because of low $\mathrm{S} / \mathrm{N}$, reinforcing the suggestion that we are seeing the entire cloud.

The innermost region of BFS15 displays the highest column density, which is $5.3 \times 10^{23} \mathrm{~cm}^{-2}$. Note that $\mathrm{S} 254 \mathrm{~S} 1$ is also comparably dense, with a column density of $4.3 \times 10^{23} \mathrm{~cm}^{-2}$. Column density maps of the other clumps such as S254N, 

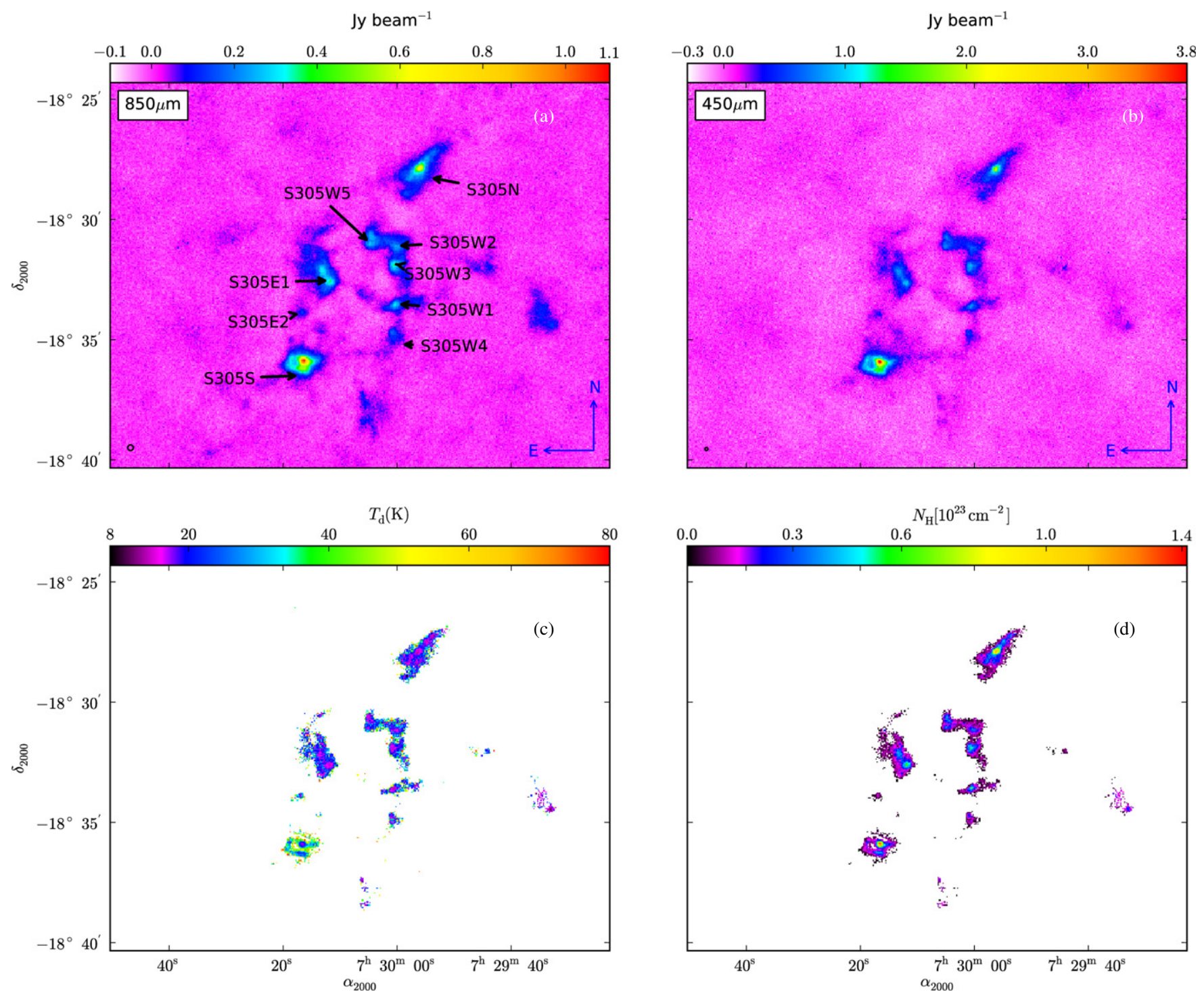

Figure 5. S305: (a) surface brightness map at $850 \mu \mathrm{m}$, (b) surface brightness map at $450 \mu \mathrm{m}$, (c) dust temperature map, and (d) column density map.

S159, S156, and the clump S305S associated with the HII region S305 also demonstrate peak column densities higher than $10^{23} \mathrm{~cm}^{-2}$ at the centers. Visual extinction values of the complexes are obtained from their derived column density maps. All the clumps, except those near S148 and S305, show peak visual extinction values above 100 . The highest peak $A_{V}(283)$ is found in the clump near BFS15. In S254N, S254S1, and S254S2, the peak $A_{V}$ values are, respectively, 199, 229, and 41. The other clumps in the sample (near HiI regions S159, S305, and S148) are characterized by peak $A_{V}$ between 63 and 198. The mean central number density, $\overline{n_{\mathrm{c}}}$ of each clump, is the average number density along the line of sight, obtained from the peak column density divided by the size of each clump. The mean central density of the clumps is typically about $8 \times$ $10^{4} \mathrm{~cm}^{-3}$. However, the central densities of the clumps range over an order of magnitude, from $10^{4}$ to $10^{5} \mathrm{~cm}^{-3}$. The value of $\overline{n_{\mathrm{c}}}$ is lower than the true central density, as it is an average that includes the lower density outer parts of the clump along the line of sight.

The derived clump properties are listed in Table 3. The first column gives the source names. The second, third, and fourth columns list the peak values of the optical depth, column density,
Table 3

Properties of the Clumps

\begin{tabular}{|c|c|c|c|c|c|c|}
\hline Source & $\tau_{850}$ & $\begin{array}{c}N_{\mathrm{H}} \\
\left(10^{23} \mathrm{~cm}^{-2}\right)\end{array}$ & $\begin{array}{c}A_{V} \\
(\mathrm{mag})\end{array}$ & $\begin{array}{l}\text { Diameter } \\
\text { (pc) }\end{array}$ & $\begin{array}{c}\overline{n_{\mathrm{c}}} \\
\left(\mathrm{cm}^{-3}\right)\end{array}$ & $\begin{array}{l}T_{\mathrm{cd}} \\
(\mathrm{K})\end{array}$ \\
\hline $\mathrm{S} 148^{\mathrm{a}}$ & $6.3 \times 10^{-3}$ & 2.0 & 86.1 & 3.56 & $1.5 \times 10^{4}$ & \\
\hline S156 & $9.9 \times 10^{-3}$ & 2.6 & 136 & 3.06 & $6.1 \times 10^{4}$ & 11.5 \\
\hline BFS15 & 0.02 & 5.3 & 283 & 1.80 & $1.6 \times 10^{5}$ & 9.5 \\
\hline S159 & 0.01 & 3.7 & 198 & 1.62 & $7.4 \times 10^{4}$ & 14.3 \\
\hline S254N & 0.01 & 3.7 & 199 & 0.76 & $1.3 \times 10^{5}$ & 24.6 \\
\hline S254S1 & 0.02 & 4.3 & 229 & 0.90 & $1.8 \times 10^{5}$ & 23.4 \\
\hline $\mathrm{S} 254 \mathrm{~S} 2$ & $3.0 \times 10^{-3}$ & 0.8 & 41 & 0.51 & $4.9 \times 10^{4}$ & 28.6 \\
\hline S305N & $4.6 \times 10^{-3}$ & 1.2 & 63 & 1.62 & $2.3 \times 10^{4}$ & 13.7 \\
\hline S305S & $5.4 \times 10^{-3}$ & 1.4 & 73 & 2.31 & $2.0 \times 10^{4}$ & 16.7 \\
\hline
\end{tabular}

Notes. Column 1 lists the clump names associated with the Galactic $\mathrm{H}$ II regions. Columns $2-4$ list the peak values of the optical depth, column density, and visual extinction (using a $\beta$ of 2 ) detected toward the centers of the respective clumps. The $850 \mu \mathrm{m}$ size, the central number densities, and the central dust temperatures (for $\beta=2$ ) of the clumps are listed in the fifth, sixth, and seventh columns, respectively.

${ }^{a}$ By assuming a constant temperature of $10 \mathrm{~K}$ throughout the cloud. 
Table 4

Properties of the Clumps Using $\beta=1.5$

\begin{tabular}{lccccc}
\hline \hline Source & $\tau_{850}$ & $\begin{array}{c}N_{\mathrm{H}} \\
\left(10^{23} \mathrm{~cm}^{-2}\right)\end{array}$ & $\begin{array}{c}A_{V} \\
(\mathrm{mag})\end{array}$ & $\begin{array}{c}\overline{n_{\mathrm{c}}} \\
\left(\mathrm{cm}^{-3}\right)\end{array}$ & $\begin{array}{c}T_{\mathrm{cd}} \\
(\mathrm{K})\end{array}$ \\
\hline S148 & $\ldots$ & $\ldots$ & $\ldots$ & $\ldots$ & $\ldots$ \\
S156 & $5.1 \times 10^{-3}$ & 1.3 & 70 & $3.1 \times 10^{4}$ & 16.9 \\
BFS15 & 0.01 & 2.9 & 154 & $8.9 \times 10^{4}$ & 12.7 \\
S159 & $6.2 \times 10^{-3}$ & 1.6 & 85 & $3.2 \times 10^{4}$ & 24.9 \\
S254N & $\ldots$ & $\ldots$ & $\ldots$ & $\ldots$ & $\ldots$ \\
S254S1 & $\ldots$ & $\ldots$ & $\ldots$ & $\ldots$ & $\ldots$ \\
S254S2 & $\ldots$ & $\ldots$ & $\ldots$ & $\ldots$ & $\ldots$ \\
S305N & $2.0 \times 10^{-3}$ & 0.52 & 28 & $1.0 \times 10^{4}$ & 23.1 \\
S305S & $1.9 \times 10^{-3}$ & 0.48 & 26 & $6.8 \times 10^{3}$ & 35.1 \\
\hline
\end{tabular}

Notes. Column 1 lists the clump names associated with the Galactic $\mathrm{H}$ II regions. Columns 2-4 list the peak values of the optical depth, column density, and visual extinction detected toward the centers of the respective clumps, respectively. The central number densities and central dust temperatures of the clumps are listed in the fifth and sixth columns, respectively.

Table 5

Properties of the Clumps Using $\beta=2.5$

\begin{tabular}{lccccr}
\hline \hline Source & $\tau_{850}$ & $\begin{array}{c}N_{\mathrm{H}} \\
\left(10^{23} \mathrm{~cm}^{-2}\right)\end{array}$ & $\begin{array}{c}A_{V} \\
(\mathrm{mag})\end{array}$ & $\begin{array}{c}\overline{n_{\mathrm{c}}} \\
\left(\mathrm{cm}^{-3}\right)\end{array}$ & $\begin{array}{r}T_{\mathrm{cd}} \\
(\mathrm{K})\end{array}$ \\
\hline S148 & $\ldots$ & $\ldots$ & $\ldots$ & $\ldots$ & $\ldots$ \\
S156 & 0.02 & 4.3 & 232 & $1.0 \times 10^{5}$ & 8.9 \\
BFS15 & 0.03 & 8.8 & 469 & $2.7 \times 10^{5}$ & 7.7 \\
S159 & 0.03 & 6.7 & 360 & $1.3 \times 10^{5}$ & 10.3 \\
S254N & 0.03 & 8.7 & 462 & $3.1 \times 10^{5}$ & 14.2 \\
S254S1 & 0.04 & 9.7 & 518 & $4.2 \times 10^{5}$ & 13.8 \\
S254S2 & $7.6 \times 10^{-3}$ & 1.9 & 104 & $1.2 \times 10^{5}$ & 15.3 \\
S305N & $8.2 \times 10^{-3}$ & 2.1 & 113 & $4.2 \times 10^{4}$ & 10.1 \\
S305S & 0.01 & 2.7 & 142 & $3.7 \times 10^{4}$ & 11.4 \\
\hline
\end{tabular}

Notes. Column 1 lists the clump names associated with the Galactic H II regions. Columns 2-4 list the peak values of the optical depth, column density, and visual extinction detected toward the centers of the respective clumps, respectively. The central number densities and central dust temperatures of the clumps are listed in the fifth and sixth columns, respectively.

and visual extinction at the centers of the clumps using the $850 \mu \mathrm{m}$ data, respectively. The size of the clump at $850 \mu \mathrm{m}$ and the average central number density values $\left(\overline{n_{c}}\right)$ are given in the fifth and sixth columns, respectively. The last column provides the values of the central dust temperatures of the clumps. Uncertainties in the diameter and number densities of the clumps are about $18 \%$ (due to uncertainties in the cloud distances) and not given in the table. Additional calibration errors of $10 \%$ at 450 and $5 \%$ at $850 \mu \mathrm{m}$ in the SCUBA-2 flux densities also contribute $11 \%$ uncertainties to the values of $\tau_{850}$, $N_{\mathrm{H}}, A_{V}, \overline{n_{\mathrm{c}}}$, and $T_{\mathrm{cd}}$ (not given in the table). These calculations were repeated for $\beta=1.5$ (see Table 4) and for $\beta=2.5$ (see Table 5).

\subsubsection{Gas Masses from Dust Emission}

The gas masses are derived by summing up all the pixel values from the column density maps of the clouds, which are generated from the $850 \mu \mathrm{m}$ dust emission maps, employing a dust temperature distribution assuming a value of $\beta$, i.e., $M_{\text {gas }}=\mu m_{\mathrm{H}} N_{\mathrm{H}} A$, where $N_{\mathrm{H}}$ is the total hydrogen column density in $\mathrm{cm}^{-2}$ and $A$ is the pixel area in $\mathrm{cm}^{2}$. The gas masses of the clouds are listed in Table 6. The first column lists the cloud names. The second, third, and fourth columns list the gas masses corresponding to $\beta$ being $2,1.5$, and 2.5 , respectively.
Table 6

Cloud Masses

\begin{tabular}{|c|c|c|c|}
\hline Source & $\begin{array}{c}M_{\text {gas }}\left(M_{\odot}\right) \\
\beta=2\end{array}$ & $\begin{array}{c}M_{\text {gas }}\left(M_{\odot}\right) \\
\beta=1.5\end{array}$ & $\begin{array}{c}M_{\text {gas }}\left(M_{\odot}\right) \\
\beta=2.5\end{array}$ \\
\hline $\mathrm{S} 148^{\mathrm{a}}$ & $(7.3 \pm 1.3) \times 10^{3}$ & $\ldots$ & $\ldots$ \\
\hline S156 & $(9.1 \pm 1.0) \times 10^{2}$ & $\left(3.1 \pm 0.3 \times 10^{2}\right.$ & $(1.7 \pm 0.4) \times 10^{3}$ \\
\hline BFS15 & $(9.9 \pm 0.6) \times 10^{2}$ & $\left(4.0 \pm 0.2 \times 10^{2}\right.$ & $(1.6 \pm 0.2) \times 10^{3}$ \\
\hline S156NE & $11 \pm 4$ & $\ldots$ & $(0.6 \pm 0.2) \times 10^{2}$ \\
\hline S159 & $(8.9 \pm 0.6) \times 10^{2}$ & $(1.0 \pm 0.4) \times 10^{2}$ & $(2.3 \pm 0.1) \times 10^{3}$ \\
\hline S159C & $(1.1 \pm 0.1) \times 10^{3}$ & $(1.7 \pm 0.5) \times 10^{2}$ & $(2.6 \pm 0.1) \times 10^{3}$ \\
\hline S254N & $(1.3 \pm 0.4) \times 10^{2}$ & $\ldots$ & $(6.6 \pm 1.9) \times 10^{2}$ \\
\hline $\mathrm{S} 254 \mathrm{~S} 1$ & $(1.1 \pm 0.3) \times 10^{2}$ & $\ldots$ & $(6.0 \pm 1.2) \times 10^{2}$ \\
\hline $\mathrm{S} 254 \mathrm{~S} 2$ & $19 \pm 7$ & $\ldots$ & $(1.3 \pm 0.6) \times 10^{2}$ \\
\hline S254N and S & $(2.8 \pm 0.5) \times 10^{2}$ & $\ldots$ & $(1.5 \pm 0.2) \times 10^{3}$ \\
\hline $\mathrm{S} 254 \mathrm{C}$ & $(4.6 \pm 0.7) \times 10^{2}$ & $\ldots$ & $(1.9 \pm 0.3) \times 10^{3}$ \\
\hline $\mathrm{S} 305 \mathrm{~N}$ & $(8.9 \pm 4.0) \times 10^{2}$ & $(2.3 \pm 1.0) \times 10^{2}$ & $(1.8 \pm 0.9) \times 10^{3}$ \\
\hline S305W1 & $(1.7 \pm 0.6) \times 10^{2}$ & $48 \pm 7$ & $(3.8 \pm 1.5) \times 10^{2}$ \\
\hline S305W2 & $(2.0 \pm 0.9) \times 10^{2}$ & $41 \pm 9$ & $(4.5 \pm 2.4) \times 10^{2}$ \\
\hline S305W3 & $(2.6 \pm 1.1) \times 10^{2}$ & $71 \pm 17$ & $(5.1 \pm 2.7) \times 10^{2}$ \\
\hline S305W4 & $(0.7 \pm 0.2) \times 10^{2}$ & $\leqslant 18$ & $(1.5 \pm 0.6) \times 10^{2}$ \\
\hline S305W5 & $(2.4 \pm 0.6) \times 10^{2}$ & $57 \pm 8$ & $(5.4 \pm 1.5) \times 10^{2}$ \\
\hline S305E1 & $(7.3 \pm 2.3) \times 10^{2}$ & $(1.6 \pm 0.4) \times 10^{2}$ & $(1.5 \pm 0.6) \times 10^{3}$ \\
\hline S305S & $(5.6 \pm 2.1) \times 10^{2}$ & $\leqslant 37$ & $(1.7 \pm 0.6) \times 10^{3}$ \\
\hline S305E2 & $15 \pm 4$ & $\ldots$ & $39 \pm 14$ \\
\hline S305C & $(3.1 \pm 0.5) \times 10^{3}$ & $\leqslant 6.6 \times 10^{2}$ & $(7.1 \pm 1.3) \times 10^{3}$ \\
\hline
\end{tabular}

Note. ${ }^{\text {a }}$ By assuming a constant temperature of $10 \mathrm{~K}$ throughout the cloud.

The uncertainties in the masses are due to the cloud's shape and they far exceed the flux uncertainties. The mass uncertainties do not include the calibration uncertainties. Flux calibration uncertainties are $\sim 10 \%$ at 450 and $5 \%$ at $850 \mu \mathrm{m}$. The letter " $\mathrm{C}$ " associated with a cloud in Table 6 refers to a cloud complex: masses of the S159, S305, and S254 complexes are also included in the table. All the individual clouds in the complexes are massive, with values of gas mass ranging from $11 M_{\odot}$ to $9.9 \times$ $10^{2} M_{\odot}$. The least massive cloud is $\mathrm{S} 156 \mathrm{NE}$, with a gas mass of $11 M_{\odot}$, while the most massive one is the $\mathrm{S} 305$ complex (S305C), with a total mass of $3.1 \times 10^{3} M_{\odot}$, at least for the $\beta=2$ case. When $\beta=2.5$, the least massive cloud is S305E2 $\left(39 M_{\odot}\right)$ and the most massive one is $\operatorname{S305C}\left(3.1 \times 10^{3} M_{\odot}\right)$. However, when $\beta=1.5$, we cannot derive temperature maps of S254C, S156NE, and S305E2, and therefore masses of these clouds are not derived. The difference in mass in all three cases is because of different cloud temperatures corresponding to different values of $\beta$.

\subsection{Analysis of Radial Intensity Profiles}

Predictions of different star formation scenarios are dependent on the true density distribution of the clumps. In order to investigate this, we construct and analyze intensity profiles of the clumps and discuss their implications for the star formation process. Without doing extensive radiative transfer modeling, it is difficult to analyze the surface brightness profiles in any detail. Simple models using power-law density and temperature distributions have been used by Shirley et al. (2000). We follow their analysis technique but caution that our sample is significantly different from the Shirley et al. (2000) objects. Their objects appear to have embedded protostars. Our sample objects do not have obvious embedded protostars and are cold at their centers; thus, it is likely that they do not have any central heating sources. 

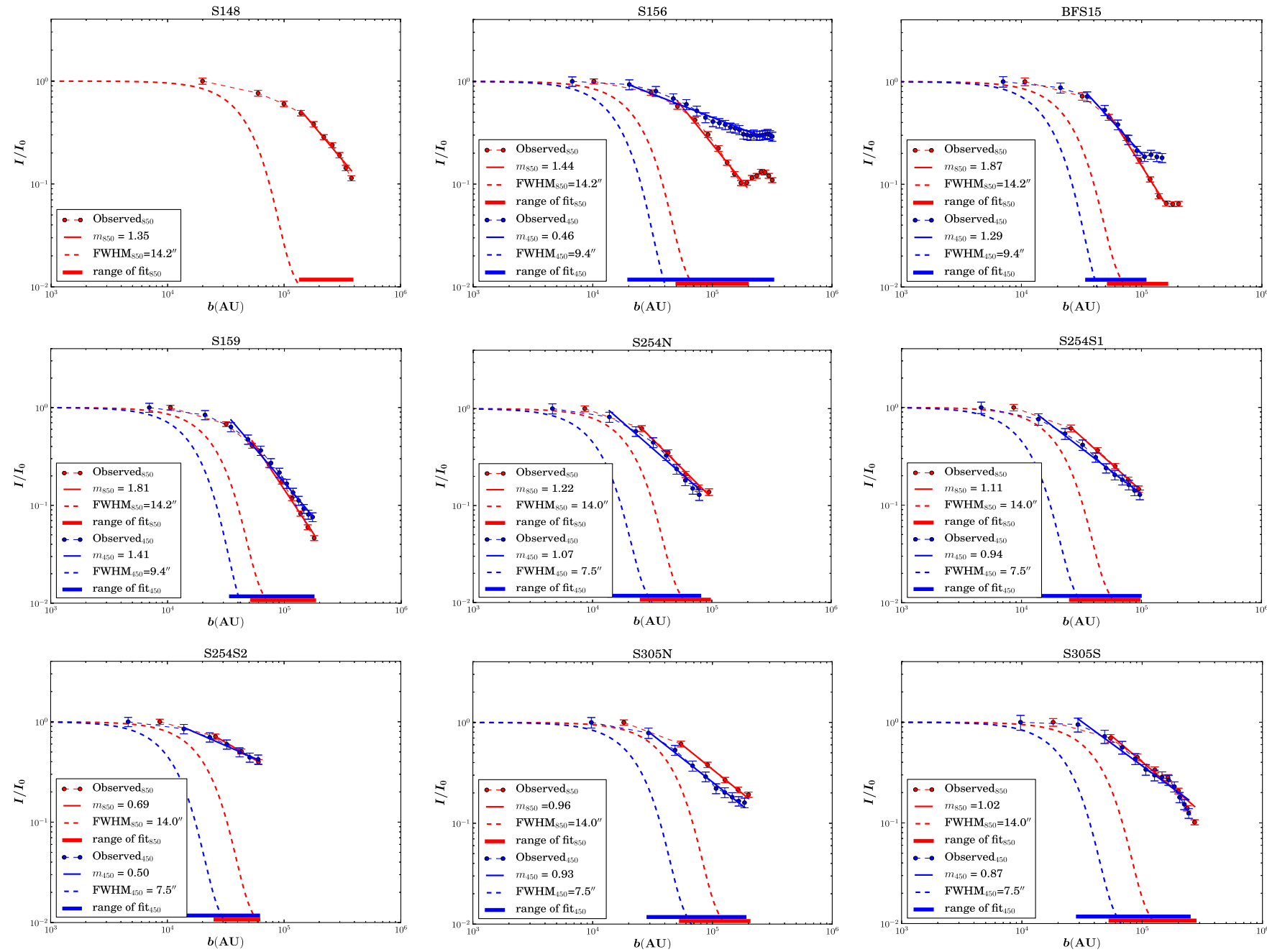

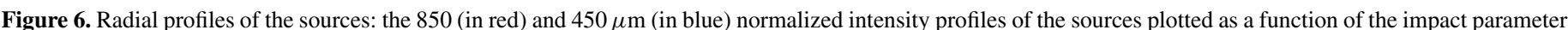

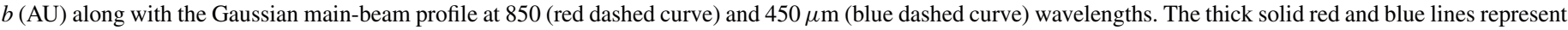
the range of the 850 and $450 \mu \mathrm{m}$ fits, respectively.

Normalized, azimuthally averaged radial profiles were constructed for each intensity map at both SCUBA-2 wavelengths. The images were binned at half the beam width to create equally spaced annuli from the peak intensity of the clumps. Uncertainties were estimated from the quadratic sum of the errors due to calibration and the deviation from the azimuthal symmetry at each radial annulus. We calculate the mean value of the intensity $I_{\nu}(b)$ normalized to the peak intensity $I_{\nu}(0)$ at each radial bin about the impact parameter $b$ (in AU). Following the technique described in Shirley et al. (2000), power-law fits were performed for all clumps using the form $I_{v}(b) / I_{v}(0)=[b / b(0)]^{-m}$, with $b(0)$ corresponding to one-quarter of the beam width. As in Shirley et al. (2000), we only fit each radial profile outside of the FWHM of the beam in each case. The intensity profiles in almost all cases are well fit by a power law (see Figure 6). We find a range of values for the power-law indices, with the average value of $1.27 \pm 0.37$ at $850 \mu \mathrm{m}$ and $0.93 \pm 0.31$ at $450 \mu \mathrm{m}$.

The SCUBA-2 beams are much larger than the inner radii of the radial profiles shown in Figure 6. Typical physical resolution is greater than $\sim 0.1 \mathrm{pc}$, or $\sim 2 \times 10^{4} \mathrm{AU}$, which is the $450 \mu \mathrm{m}$ SCUBA-2 beam width of $7^{\prime \prime} .5$ at a distance of $2.46 \mathrm{kpc}$ - the distance to the closest object in our sample. The average value of $m$, obtained by combining the slopes at 850 and $450 \mu \mathrm{m}$ wavelengths, is $1.10 \pm 0.34$. This value agrees well with the outer slope of 1 in the intensity distribution of isolated lowmass pre-protostellar cores studied by Ward-Thompson et al. (1994). Our average value of $m$ is slightly flatter than the mean slope of $1.48 \pm 0.35$ obtained for low-mass Class 0/I sources by Shirley et al. (2000) and is consistent with the mean inner power-law intensity index of 1.2 found in massive star-forming regions by Beuther et al. (2002).

If we consider an optically thin uniform opacity spherically symmetric clump with $I_{v}(b) \propto b^{-m}$ and dust temperature $T_{\mathrm{d}}(r) \propto r^{-q}$, then the density profile of the clump $\rho(r)$ will follow $r^{-p}$, where the power-law indices can be related as $m=p+q-1$ (Adams 1991). The slopes of the radial temperature profiles (corresponding to $\beta=2$ ) of our objects, calculated from the dust temperature maps, are used to calculate the density power-law index $p$. Since we found an average $q$ of $-0.26 \pm 0.10$ in five of our clumps, the average value of $m$ therefore translates into an average $p$ of $2.36 \pm 0.35$. All the uncertainty values represent uncertainties due to the standard deviation of the sample.

The mean value of $p$ from our study is consistent with the mean value of the density power-law index $(p=2.15 \pm 0.35)$ corresponding to a $\beta$ of 2 in low-mass Class $0 / \mathrm{I}$ sources (Shirley et al. 2000). However, the cores in the study of Shirley et al. (2000) are centrally heated, whereas all the clumps in 
our study have decreasing temperatures toward the centers. Our value of $p$ is steeper than the density power-law index, obtained using one-dimensional (1D) radiative transfer codes, of $1.8 \pm 0.4$ (Mueller et al. 2002-in dense cores associated with massive star-forming regions) and $1.3 \pm 0.4$ (Williams et al. 2005 - in a sample of high-mass protostellar objects). The $p$ values obtained using power-law fits such as $1.6 \pm 0.5$ (Beuther et al. 2002-in a large sample of massive cores in the earliest stages of evolution) and $1.6 \pm 0.3$ (Pirogov 2009-in highmass star-forming regions of the southern hemisphere) are also shallower than those from our study. Similarly, 1D radiative transfer studies by Jørgensen et al. (2002) on low-mass Class 0 and Class 1 sources show flatter density power-law indices in the range 1.3-1.9 $( \pm 0.2)$ within $\sim 10^{4} \mathrm{AU}$. However, all these values are dependent on the assumed values of the dust emissivity index, $\beta$.

Our power-law indices for densities are steeper than all of these results found by other authors. However, all of these other results are for cores with embedded sources, while our objects do not show any signs of embedded sources. Perhaps this difference in indices is due to this difference in the evolutionary state of the objects. Hydrostatic and hydrodynamic configurations of pressure-bounded cores have density profiles in their outer envelopes that fall off like $r^{-2}$, similar to what we have found in our studies. The study (Tafalla et al. 2002) of starless cores in low-mass star-forming regions reveals power-law indices ranging from 2 to 4 at large radii, consistent with our mean value of $p$. If our objects are truly starless, then we would expect the density structure to flatten in the inner regions. Nevertheless, our resolution (and the source distance) severely limits the ability to see this, and all the power-law structure observed in our study is typically outside of $10^{4} \mathrm{AU}$.

\subsection{Caveats}

The observed flux densities from sub-millimeter thermal emission are plagued by a number of uncertainties, which can influence the derived dust temperatures and densities. These uncertainties include possible line contamination, variations in the calibration factor, discrepancies in the adopted convolution method, and variations of the value of $\beta$. In addition, the assumptions used in the analysis of radial intensity profiles are sensitive to the Rayleigh-Jeans limit.

Spectral line contamination in the SCUBA-2 bolometer bandpasses may be a significant source of uncertainty in the extracted physical properties. Abundant molecules such as $\mathrm{CO}$ and its isotopomers embedded in dust clouds are one of the primary sources of dust continuum pollutants at submillimeter wavelengths (Gordon 1995; Papadopoulos \& Allen 2000; Seaquist et al. 2004). The major contributor to line emission in the $850 \mu \mathrm{m}$ band is expected to be the $\mathrm{CO}(3-2)$ line at $345 \mathrm{GHz}$. Methanol and $\mathrm{SO}_{2}$ are likely to be significant contributors to the continuum emission-although much weaker than the $\mathrm{CO}$ lines, these molecules emit in dozens or even hundreds of separate lines within the bandpass. We can estimate the effect on our results if there is a significant amount of line emission in our $850 \mu \mathrm{m}$ images but none in our $450 \mu \mathrm{m}$ images. This is effectively a "worst" case, because the presence of line emission in the $850 \mu \mathrm{m}$ image would cause us to infer a lower temperature, while such emission in the $450 \mu \mathrm{m}$ image would move our calculated temperatures higher. However, it is possible that in our clouds the line emission is very weak, because the regions are very cold. For example, the $\mathrm{CO}$ emission in the $850 \mu \mathrm{m}$ band is the $J=3 \rightarrow 2$ transition, which is bright in warm regions. We have $\mathrm{CO}(3-2)$ data for some of our objects, and as we discuss below, we find that this is not an important effect in the denser, colder centers of these clouds. The CO has a significant effect on the observed fluxes in the hot outer edges of most of these clouds.

We have obtained $\mathrm{CO}(3-2)$ data from the JCMT archives $^{11}$ for four of our objects: S156, S159, S254, and S305S. The $\mathrm{CO}(3-2)$ contribution to the SCUBA-2 $850 \mu \mathrm{m}$ dust continuum emission is found to be below $\sim 2 \%-3 \%$ toward the centers of S159, S254N, and S254S, and $\sim 10 \%$ at the outermost borders of the clumps. The extended envelopes of the S159 cloud exhibit higher contributions from the $\mathrm{CO}(3-2)$ line: $\sim 15 \%-45 \%$ for the northwest and east, $\sim 40 \%-90 \%$ for the northeast envelope. A few pixels at the northwest part of the central S254 cloud show dominant $\mathrm{CO}(3-2)$ line contribution, $\sim 30 \%-60 \%$. Overall, the $\mathrm{CO}(3-2)$ line contributes little to the bulk of the S254 cloud. Contamination under $\sim 15 \%$ toward the centers and $<40 \%$ toward the outer borders of the clumps S156 and S305S is observed. The $\mathrm{CO}(3-2)$ line emission contributed $\sim 60 \%-90 \%$ of the SCUBA-2 $850 \mu \mathrm{m}$ flux at some parts of the extended envelope of S156. Recent studies on the Perseus B1 clump by Sadavoy et al. (2013) found that $\mathrm{CO}(3-2)$ line emission contributed only less than $15 \%$ of the $850 \mu \mathrm{m}$ flux on the internally heated regions and less than $1 \%$ of the $850 \mu \mathrm{m}$ flux on the colder regions of the B1, with the most significant contribution, $\sim 90 \%$, at the outflow positions. Studies on a number of nebulae in the Perseus and Orion cloud complexes by Drabek et al. (2012) confirmed less than 20\% contribution from the $\mathrm{CO}(3-2)$ line emission on the SCUBA-2 $850 \mu \mathrm{m}$ flux in regions without outflows and an insignificant contribution from $\mathrm{CO}(6-5)$ lines at $450 \mu \mathrm{m}$ dust emission.

The removal of line-contaminated flux densities from the SCUBA-2 $850 \mu \mathrm{m}$ data has only a minor effect on the dust temperatures in the dense clumps (see Figure 7(a)): subtraction of the observed S159 CO(3-2) data from the $850 \mu \mathrm{m}$ SCUBA-2 data barely changed the central dust temperatures, although it slightly increased the outer temperatures (Figure 7(a)-blue curve). If there is a higher contribution from $\mathrm{CO}$ or from other molecular lines within this band (for example, $\sim 30 \%$ ), then the clump temperatures alter considerably: removal of an assumed $30 \%$ line contamination increased the temperatures by around $93 \%$ at the center. However, due to very high $450 / 850$ flux ratio at the edges, the temperatures at the clump edges could not be evaluated. Elimination of the line contribution to the continuum (using the observed $\mathrm{CO}$ data) did not change the density powerlaw index. Although the $\mathrm{CO}(3-2)$ emission does not affect the main results of this paper (very cold, dense clumps), it does cause us to slightly underestimate the temperature at the edges of the clouds surrounding the clumps. A more detailed analysis of the $\mathrm{CO}$ emission is underway and will appear in a future paper.

Small variations in the adopted value of the FCF have a negligible effect on the results. For example, employing the standard value of FCF to calibrate the $\mathrm{S} 156$ map field, by raising the value of FCF by $\sim 2 \%$ at 850 and $\sim 7 \%$ at 450 , caused only $\sim 4 \%-5 \%$ variations in the central dust temperatures and $\sim 7 \%$ variations in the peak dust column densities of the clouds.

Since the $850 \mu \mathrm{m}$ beam solid angle is larger due to the error beam pattern than just the main-beam solid angle and there is large difference in the side-lobe patterns between 450 and $850 \mu \mathrm{m}$, convolving the $450 \mu \mathrm{m}$ SCUBA-2 map to the $850 \mu \mathrm{m}$

\footnotetext{
11 http://www.cadc-ccda.hia-iha.nrc-cnrc.gc.ca/jcmt/
} 

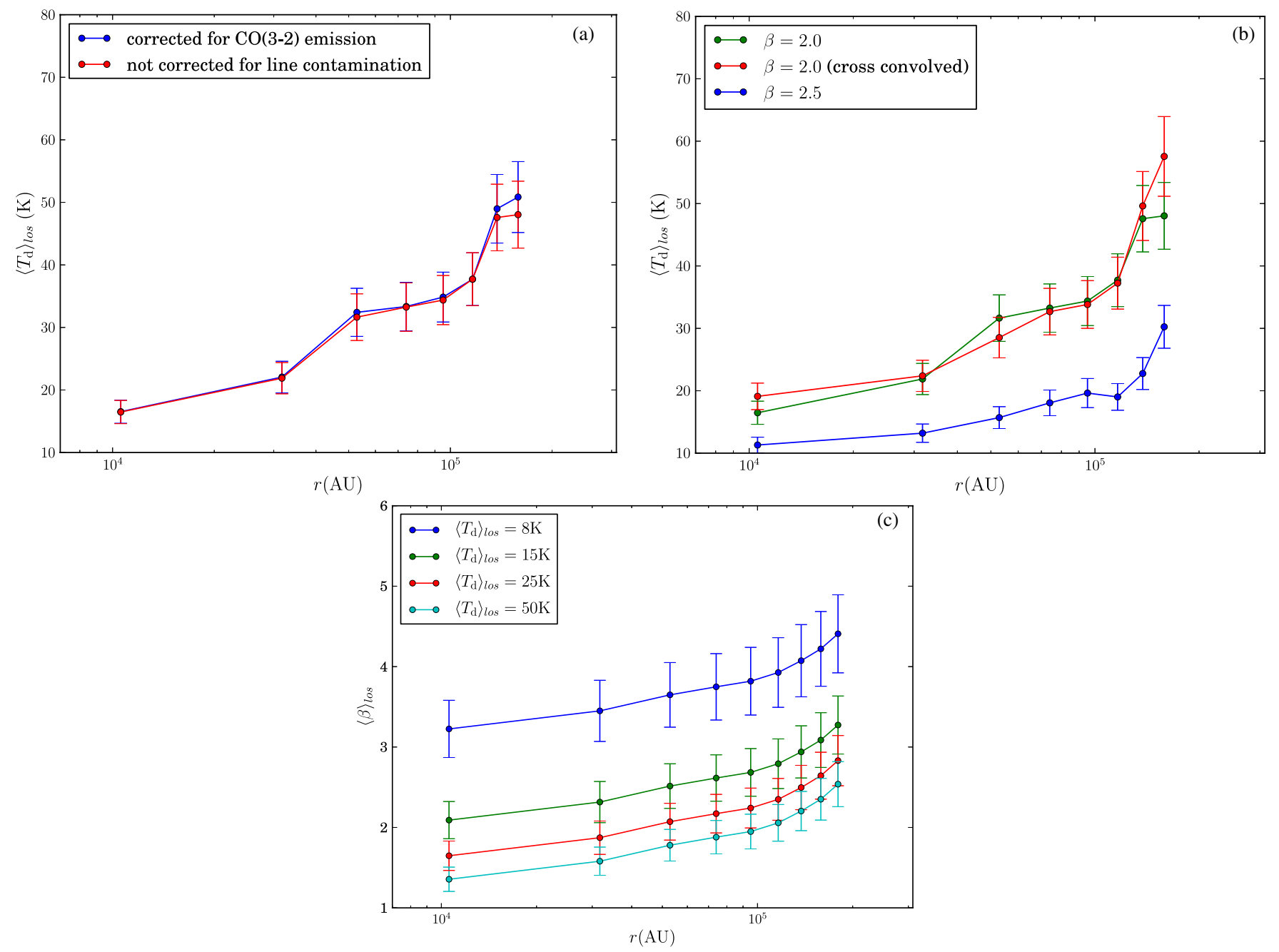

Figure 7. (a) Line-of-sight averaged dust temperature $\left(\left\langle T_{\mathrm{d}}\right\rangle_{\mathrm{los}}\right)$ profiles of the cloud associated with $\mathrm{S} 159$, with (red curve) and without (blue curve) line contamination at $850 \mu \mathrm{m}$. The blue curve represents temperature obtained from the data corrected for $\mathrm{CO}(3-2)$ emission at $850 \mu \mathrm{m}$, using a $\beta$ of 2. (b) Variations in the $\left\langle T_{\mathrm{d}}\right\rangle_{\text {los }}$ profile with different assumed values of $\beta$. The red curve represents the temperature obtained from the 450/850 ratio map, using a $\beta$ of 2 , after doing cross-convolution

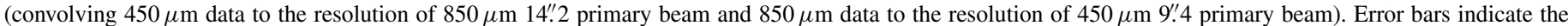
uncertainty in the temperature due to a combination of calibration and the error in the mean of the temperature distribution in each radial annulus. (c) Variations in $\beta$ corresponding to different possible values of $\left\langle T_{\mathrm{d}}\right\rangle_{\text {los }}$ from $8 \mathrm{~K}$ to $50 \mathrm{~K}$. Error bars indicate the uncertainty in $\beta$ due to a combination of calibration and the error in mean of the $\beta$ distribution in each radial annulus.

resolution of the JCMT beam, to roughly match the Gaussian main beams at both wavelengths, may lead to some uncertainties in the result. We therefore performed a cross-convolution on one of the clouds (S159), by convolving $450 \mu \mathrm{m}$ data to the resolution of $850 \mu \mathrm{m} \mathrm{14} .2$ primary beam and $850 \mu \mathrm{m}$ data to the resolution of $450 \mu \mathrm{m} \mathrm{9.'4} \mathrm{primary} \mathrm{beam} \mathrm{as} \mathrm{described} \mathrm{in}$ Hatchell et al. (2013). The cross-convolution method raised the value of the dust temperature at the center of S159 by around $3 \mathrm{~K}$. However, this method did not make a large increase in the temperature, and all the values obtained from this method are within the uncertainty limits of the temperatures obtained by the method used in this paper (see Figure 7(b)).

Variations in the $\left\langle T_{\mathrm{d}}\right\rangle_{\text {los }}$ profiles of the clump close to S159, corresponding to different values of $\beta$, are displayed in Figure 7(b). Since there is some evidence of $\beta>2$ toward low-mass star-forming regions (Schnee et al. 2010; Shirley et al. 2011), we also explored the possibility of $\beta=2.5$ in our calculations. As the value of $\beta$ increases from 2.0 to 2.5 , the $\left\langle T_{\mathrm{d}}\right\rangle_{\text {los }}$ profile shifts downward, denoting an anticorrelation between temperature and values of $\beta$, a very well known effect, discussed by many authors. Our determination of the temperatures depends on what value of $\beta$ we use in the calculations. The central clump temperatures shift from $\sim 10 \mathrm{~K}$ to $14 \mathrm{~K}$ as we decrease the value of $\beta$ from 2.5 to 2.0 , indicating cold central regions even in the presence of lower $\beta$. Similarly, between $\beta$ of 1.5 and 2.5, Sadavoy et al. (2013) found $\sim 4 \mathrm{~K}$ variation in the dust temperature toward the B1-a core. However, when $\beta$ is 1.5 , the central clump temperature of S159 is $\sim 25 \mathrm{~K}$, but we are unable to plot a temperature profile due to high values of the 450/850 ratio toward the outer layers. The change in $\beta$ barely affects the density power-law index; for example, as we increase the value of $\beta$ from 2 to 2.5 , the average value of $p$ increases by merely $1 \%$. The clouds show another conspicuous feature: regions of lower temperatures correspond to higher column densities if $\beta$ is uniform throughout the cloud. This feature might imply that the dust grains in the densest regions of the clouds are effectively shielded from the stellar radiation field. As a consequence, grains in these regions are more likely to coalesce than shatter due to lower average kinetic energy (Chokshi et al. 1993).

Using the emission from the two SCUBA-2 wavelengths, we computed the value of the dust emissivity index in each pixel 
by assuming a constant line-of-sight averaged dust temperature of $8 \mathrm{~K}, 15 \mathrm{~K}, 25 \mathrm{~K}$, and $50 \mathrm{~K}$ throughout the cloud (see Figure $7(\mathrm{c})$ ). The assumed temperatures are considered to be typical temperatures that can be found in different radial layers of the cold GMCs. A radial variation of $\beta$ is observed in all the complexes at all selected temperatures, with low values (e.g., $\beta \sim 1.3$ at $\left.\left\langle T_{\mathrm{d}}\right\rangle_{\text {los }}=50 \mathrm{~K}\right)$ and high values $(\beta \approx 3.2$ at $\left.\left\langle T_{\mathrm{d}}\right\rangle_{\text {los }}=8 \mathrm{~K}\right)$ toward the center of S159. Increasing the assumed temperature from $8 \mathrm{~K}$ to $50 \mathrm{~K}$ produces on average a decrease in $\beta$ by $59 \%$ toward the center of S159. Much lower values of $\beta$ $(\sim 0.5)$ are observed toward the center of the southwest fragment cloud in the $\mathrm{S} 159$ complex when $\left\langle T_{\mathrm{d}}\right\rangle_{\text {los }}=50 \mathrm{~K}$. Note that the lowest value of $\beta$ detected in the direction of the isolated lowmass star-forming core TMC-1C's center (Schnee \& Goodman $2005)$ is $\sim 1.7$. At each $\left\langle T_{\mathrm{d}}\right\rangle_{\text {los }}$, one can see lower values of $\langle\beta\rangle_{\text {los }}$ toward the centers of the clouds. Calculating variations in $\left\langle T_{\mathrm{d}}\right\rangle_{\text {los }}$ by assuming $\langle\beta\rangle_{\text {los }} \sim$ constant is probably much closer to reality than calculating variations in $\langle\beta\rangle_{\text {los }}$ by assuming that $\left\langle T_{\mathrm{d}}\right\rangle_{\text {los }}$ is constant because the range of $\langle\beta\rangle_{\text {los }}$ in the latter case is far outside anything actually measured at the sub-millimeter regime (e.g., up to 3.2 in the centers).

The assumption that $m=p+q-1$, used in the analysis of radial intensity profiles of the clumps, is valid only in the Rayleigh-Jeans limit, where $(h v / k) \ll T$. At temperatures below $17 \mathrm{~K}$ at $850 \mu \mathrm{m}$, the Rayleigh-Jeans law breaks down and the interiors of our clumps, wherever $T_{\mathrm{d}}$ is less than $17 \mathrm{~K}$, will not satisfy power-law equations. To correct for this, one needs to go back to the Adams (1991) analysis and then change it to account for the low temperatures. However, this will not allow us to directly compare the results to Shirley et al. (2000), Beuther et al. (2002), and Pirogov (2009), as we have done in this paper. Furthermore, modifying the expressions of Adams (1991) is mathematically too challenging for the current paper.

\section{DISCUSSION}

Stars usually form in locations of cold, dense molecular gas at high densities, over $10^{4} \mathrm{~cm}^{-3}$, and GMCs are ideal sites for such dense concentrations of gas. However, the star formation efficiency, defined as the star formation rate per unit mass of gas, in GMCs is found to be very low, less than 3\% (Myers et al. 1986; Lada 1987), compared to the star formation efficiency in low-mass star-forming regions. The efficiency of isolated star formation is $9 \%-15 \%$ (Swift \& Welch 2008) and as high as $30 \%-50 \%$ (Matzner \& McKee 2000) for clustered low-mass star formation. However, in regions of protostellar outflows, the lowmass star formation efficiency can be $25 \%-70 \%$ (Matzner \& McKee 2000). Analyzing the clumps within GMCs is therefore important to better understand the low efficiency of their star formation.

The temperatures of the clouds determine the initial stages of star formation. To verify the conditions in the clumps, we calculated the Jeans mass of the clumps (for $\beta=1.5,2$, and 2.5) using central average number densities, assuming that the gas and the dust temperatures are coupled inside the clumps. At regions of high density, higher than $\approx 10^{4} \mathrm{~cm}^{-3}$, the gas and the dust temperatures share the same value (Galli et al. 2002; Goldsmith 2001). The derived Jeans masses of the clumps are significantly smaller than the mass of the clumps calculated from the dust emission, suggesting that gravitational attraction overwhelms the thermal pressure, and in the absence of other non-thermal support mechanisms, the clumps will undergo a contraction that in turn is responsible for a probable eventual collapse. Moreover, there could be cores within these clumps that are collapsing (and still starless); their existence is unknown without higher resolution observations. The support mechanisms may include turbulence or magnetic fields. Ammonia-line observations (to test for turbulence) or observations of sub-millimeter polarization (to look for ordered magnetic fields) would aid in our understanding of how these clumps survive without collapse.

We cannot constrain the values of $\beta$ and temperature simultaneously using just two wavelengths. We therefore assumed a constant $\beta$ to calculate the temperature variations in our cloud samples. However, $\beta$ may not be uniform in real clouds. Data from shorter Herschel wavelengths (PACS 160 and SPIRE data) with SCUBA-2 data may better constrain the values of the dust temperatures because we can simultaneously calculate temperature and $\beta$. However, we have not observed the clouds in Herschel bands, and therefore we calculated the physical properties of the clouds with a $\beta$ of $1.5,2$, and 2.5 to get a possible range of physical values. The inclusion of filtered longwavelength SCUBA-2 data to Herschel data has been found to increase the reliability of the temperature and $\beta$ calculation (Sadavoy et al. 2013). Herschel observations are more sensitive to larger scale structures, but since such structures contribute to both 450 and $850 \mu \mathrm{m}$ emission, it is not clear to what extent this affects our results. In any case, our focus is on the smaller scale structures - the dense clumps-and in order to get color temperatures for these, it would be necessary to remove the larger scale structure, which SCUBA-2 already does for us.

We have found cold dust, $9.5-28.6 \mathrm{~K}$ for $\beta$ of 2 and $7.7-15.3 \mathrm{~K}$ for $\beta$ of 2.5 , near the $\mathrm{H}$ II regions considered in this study. But even with very shallow values of $\beta$, e.g., $\beta=1.5$ everywhere, we have found central temperatures ranging from $\sim 13 \mathrm{~K}$ to $35 \mathrm{~K}$ in most of the clouds. The clumps are still unstable to gravitational collapse even with such shallow values of $\beta$. All these analyses imply that our target clumps are indeed cold and that therefore there is not yet any protostar at their centers. Recent studies by Launhardt et al. (2013) have found similar observational evidence of cold dust $(8-12 \mathrm{~K})$ at the interiors, with dominant heating (14-20 K) from the interstellar radiation field (ISRF) at the outer rims, on a number of isolated low-mass starless cloud cores.

Our results of very cold dust near high-mass star-forming regions are consistent with the range of theoretical minimum temperatures found in low-mass starless cores (Evans et al. 2001). Besides dust continuum absorption of the ISRF, Evans et al. (2001) considered different heating mechanisms that will elevate the dust temperature at the center of a starless core/ clump such as cosmic rays, the effect of UV photons that are created following cosmic-ray heating, and collision with warm gas. However, note that all our clouds are farther from the Galactic center than the Evans et al. (2001) clouds-in some cases $50 \%$ farther, which means that heating by cosmic rays should be significantly less and our clouds are bigger and denser, and thus heating by ISRF and UV is also considerably less, except, most likely, in the case of the central clumps in the S254 complex.

In nearby, low-mass starless cores, the typical outer dust temperature is $\sim 20 \mathrm{~K}$ for ISRF intensity, $G_{0}, \sim$ few. Typical outer dust temperature in our clumps is $\sim 60 \mathrm{~K}$, and since (for $\beta=2$ ) outer dust temperature is $\sim G_{0}^{(1 / 6)}$, this means that $G_{0}$ is $\sim 1000$ times higher than those probed in local cores not associated with $\mathrm{H}$ II regions. This is what we expect for clumps near strong radiation fields of O/B stars (Jørgensen et al. 2006). 
Not much is known about the structure of the density distribution in high-mass clumps, although this is rigorously studied in low-mass cores. The high-mass clumps in our study possess a steepened outer region $\left(\rho \propto r^{-2.36}\right)$ for a $\beta$ of 2 , and the power-law structure observed is outside of typically 10,000 AU. The inner radial profile of our target clumps appears to be flatter than the outer envelope; however, these regions are well within the telescope beam and are unresolved. Inner flattening of the density profiles, with $\rho \propto r^{-2}$ toward the outer regions, is found to be a characteristic feature of evolving low-mass pre-stellar cores (Ward-Thompson et al. 1994, 1999; Andre et al. 1996, 2000). An isothermal low-mass spherical core that collapses to form stars follows an $r^{-2}$ density profile (Shu 1977). If high-mass star formation is a scaled-up version of low-mass star formation, the cold clumps and decreasing temperatures toward the clump centers, along with steeper outer density power laws, most likely suggest that the centers have not yet gained the density required for the formation of high-mass stars. These clumps are so massive that they may be fragmenting into multiple cores, but it will require interferometric observations to sort this out. High-resolution observations of the clumps are therefore needed for a detailed analysis of the innermost density structure of the high-mass clumps and their star formation scenarios.

The masses of the individual clouds range from around 10 to $1000 M_{\odot}$, for $\beta$ of 2 and 2.5. Three of our complexes, namely, S254 (Heyer et al. 1989), S305, and S148 (Azimlu \& Fich 2011), were studied using CO data. The mass of the S254 complex obtained from $\mathrm{CO}$ observations $\left(2.7 \times 10^{4} M_{\odot}\right)$ is higher than the value obtained from our studies $\left(4.6 \times 10^{2} M_{\odot}\right.$ $\left.(\beta=2), 1.9 \times 10^{3} M_{\odot}(\beta=2.5)\right)$, indicating that we may have underestimated the gas mass. This probably suggests that this is a complex region because there are so many $\mathrm{H}$ II regions. However, the mass of the S148 complex calculated from CO studies $\left(7.9 \times 10^{3} M_{\odot}\right)$ is close to our results $\left(7.3 \times 10^{3} M_{\odot}\right)$. Similarly, the mass of the S305 complex from CO studies $\left(3.8 \times 10^{3} M_{\odot}\right)$ is close to the mass obtained from our studies $\left(3.1 \times 10^{3} M_{\odot}(\beta=2), 7.1 \times 10^{3}(\beta=2.5)\right)$.

The masses obtained from the column density maps using the observed sub-millimeter data could be an overestimation of the true cloud masses. Recent research on star-forming clumps, using simulations that model the gravitational collapse of molecular clouds, argue that the presence of lower density materials along the sightline can result in an overestimation of the cloud mass by a factor of up to three (Ward et al. 2012). All our clouds are vulnerable to this effect, and there can thus be a factor of up to three decrease in the observed cloud masses. This effect is dependent on the mass: toward massive regions, this effect is more pronounced, due to the influence of much higher density material along the line of sight.

The high visual extinction, high mass, high column densities, and cold temperatures of our sample clumps favor an infalling state over an equilibrium state. At visual extinctions greater than 100 and column densities higher than $10^{23} \mathrm{~cm}^{-2}$ at very high masses, the cloud cores are unstable to collapse (Kauffmann $\&$ Bertoldi 2004). All the observed clumps come under the category of high-mass starless clumps (HMSCs), which by definition do not host any massive protostars at the center, and are the probable precursors of high-mass protostellar objects. HMSCs represent initial conditions of high-mass star formation and are among the least studied objects in the field of star formation. This is because these objects' peak emission falls in the sub-millimeter regime, and until recently we were unable to probe their physical properties due to the lack of sufficient instruments that operate at these wavelengths.

Very few HMSCs are known to date, and probing the properties of HMSCs is central to understanding the massive star formation process. Because these regions evolve fast, they most likely generate massive stars that live for around a million years without clearing out their dust cocoon. Most of the known HMSCs (Sridharan et al. 2005) have masses ranging from $10^{2}$ to $10^{3} M_{\odot}$ and are at multiple evolutionary stages. Additional HMSC candidates have been identified in a handful of studies over the past couple of years. Using $870 \mu \mathrm{m}$ ATLASGAL data, Tackenberg et al. (2012) identified starless clumps with masses ranging from tens to tens of thousands of solar mass, having peak column densities $>10^{23} \mathrm{~cm}^{-2}$. Analysis of IRDCs using Herschel Infrared Galactic Plane Survey (Hi-Gal) data confirmed the presence of starless cores with masses ranging from 7 to $200 M_{\odot}$ and central densities spanning $\sim 10^{3}-10^{5} \mathrm{~cm}^{-3}$ (Wilcock et al. 2012).

Typical molecular clouds of our samples possess total IR luminosities ranging from $10^{3}$ to $10^{6} L_{\odot}$, and some of the clouds are identified with young stellar populations and open stellar clusters-primarily the main-sequence stars associated with them (Azimlu 2010) — and are at multiple evolutionary stages. One of our SCUBA-2 targets, S254, is a multiple star-forming complex, and Class I and Class II young stellar objects, which represent stars with an infalling envelope and disk, respectively, are detected in the region (Chavarría et al. 2008). Thus, even in such complexes, we can find cold dust.

\section{CONCLUSION}

In this paper, we have studied molecular clouds near Galactic $\mathrm{H}$ II regions, using dust continuum observations performed using the JCMT SCUBA-2 camera. A total of five Galactic $\mathrm{H}$ II regions were targeted using SCUBA-2. The central goal of this work has been to measure the temperature and density of these clouds through the continuum emission from the dust and thereby to gain insight into the processes of massive star formation. The exteriors of these clouds are subjected to intense heating from the $\mathrm{O}$ stars powering the $\mathrm{H}$ II regions immediately adjacent to the clouds in our sample. However, these clouds are very dense, with extremely high visual extinctions from their surfaces to most of their interior volume. The dust temperature distribution in molecular clouds near Galactic $\mathrm{H}$ II regions was examined using the 450 and $850 \mu \mathrm{m} \mathrm{SCUBA-2} \mathrm{data,} \mathrm{leading} \mathrm{to} \mathrm{the} \mathrm{identification}$ of very dense clumps with cold dust, down to $8-17 \mathrm{~K}$ at the centers for emissivity indices of $\beta=2.5-1.5$, near hot starforming environments. All of the regions observed show signs of filamentary structure, although not as clearly in some cases as in others. We suspect that this is due to the lessened sensitivity to larger scale structures in these images. The dense clumps are found along the centers of these filaments in those cases where the filament is clearly seen. We find that these clumps are cold even after accounting for possible line contamination in the continuum and even if the dust emissivities are shallow. At these low temperatures longer wavelength observations, such as with SCUBA-2 at $850 \mu \mathrm{m}$, have a greater ability to separate the emission from that due to hotter regions.

The James Clerk Maxwell Telescope is operated by the Joint Astronomy Centre on behalf of the Science and Technology Facilities Council of the United Kingdom, the National Research Council of Canada, and (until 2013 March 31) the 
Netherlands Organisation for Scientific Research. Additional funds for the construction of SCUBA-2 were provided by the Canada Foundation for Innovation. We are grateful to Dr. Carolyn McCoey (University of Waterloo) for several important discussions and Dr. David Berry (JAC, Hawaii) for valuable assistance regarding SCUBA-2 data reduction. This research made use of pywcsgrid $2^{12}$ - an open-source plotting package for Python.

\section{REFERENCES}

Adams, F. C. 1991, ApJ, 382, 544

Anderson, L. D., Zavagno, A., Rodón, J. A., et al. 2010, A\&A, 518, L99

Andre, P., Ward-Thompson, D., \& Barsony, M. 2000, in Protostars and Planets

IV, ed. V. Mannings, A. P. Boss, \& S. S. Russell (Tucson, AZ: Univ. Arizona Press), 59

Andre, P., Ward-Thompson, D., \& Motte, F. 1996, A\&A, 314, 625

Azimlu, M. 2010, PhD thesis, Univ. Waterloo

Azimlu, M., \& Fich, M. 2011, AJ, 141, 123

Beckwith, S. V. W., \& Sargent, A. I. 1991, ApJ, 381, 250

Beuther, H., Schilke, P., Menten, K. M., et al. 2002, ApJ, 566, 945

Bolatto, A. D., Wolfire, M., \& Leroy, A. K. 2013, ARA\&A, 51, 207

Chapin, E. L., Berry, D. S., Gibb, A. G., et al. 2013, MNRAS, 430, 2545

Chavarría, L. A., Allen, L. E., Hora, J. L., Brunt, C. M., \& Fazio, G. G. 2008, ApJ, 682,445

Chokshi, A., Tielens, A. G. G. M., \& Hollenbach, D. 1993, ApJ, 407, 806

Crampton, D., Georgelin, Y. M., \& Georgelin, Y. P. 1978, A\&A, 66, 1

Dempsey, J. T., Friberg, P., Jenness, T., et al. 2013, MNRAS, 430, 2534

Désert, F., Macas-Prez, J. F., Mayet, F., et al. 2008, A\&A, 481, 411

Dickman, R. L. 1978, ApJS, 37, 407

Drabek, E., Hatchell, J., Friberg, P., et al. 2012, MNRAS, 426, 23

Draine, B. T. 2003, ARA\&A, 41, 241

Draine, B. T., \& Lee, H. M. 1984, ApJ, 285, 89

Dupac, X., Bernard, J., Boudet, N., et al. 2003, A\&A, 404, L11

Evans, N. J., II, Rawlings, J. M. C., Shirley, Y. L., \& Mundy, L. G. 2001, ApJ, 557,193

Galli, D., Walmsley, M., \& Gonçalves, J. 2002, A\&A, 394, 275

Giannini, T., Elia, D., Lorenzetti, D., et al. 2012, A\&A, 539, A156

Goldsmith, P. F. 2001, ApJ, 557, 736

Gordon, M. A. 1995, A\&A, 301, 853

Hatchell, J., Wilson, T., Drabek, E., et al. 2013, MNRAS, 429, L10

Heyer, M. H., Snell, R. L., Morgan, J., \& Schloerb, F. P. 1989, ApJ, 346, 220

Holland, W. S., Bintley, D., Chapin, E. L., et al. 2013, MNRAS, 430, 2513

Jenness, T., Berry, D., Chapin, E., et al. 2011, in ASP Conf. Ser. 442, Astronomical Data Analysis Software and Systems XX, ed. I. N. Evans, A. Accomazzi, D. J. Mink, \& A. H. Rots (San Francisco, CA: ASP), 281

Jenness, T., Cavanagh, B., Economou, F., \& Berry, D. S. 2008, in ASP Conf. Ser. 394, Astronomical Data Analysis Software and Systems XVII, ed. R. W. Argyle, P. S. Bunclark, \& J. R. Lewis (San Francisco, CA: ASP), 565

Jenness, T., \& Economou, F. 1999, in ASP Conf. Ser. 172, Astronomical Data Analysis Software and Systems VIII, ed. D. M. Mehringer, R. L. Plante, \& D. A. Roberts (San Francisco, CA: ASP), 171

Johnstone, D., \& Bally, J. 2006, ApJ, 653, 383

Johnstone, D., Fich, M., Mitchell, G. F., \& Moriarty-Schieven, G. 2001, ApJ, 559,307

Johnstone, D., Matthews, H., \& Mitchell, G. F. 2006, ApJ, 639, 259

Jørgensen, J. K., Johnstone, D., van Dishoeck, E. F., \& Doty, S. D. 2006, A\&A, 449,609

Jørgensen, J. K., Schöier, F. L., \& van Dishoeck, E. F. 2002, A\&A, 389, 908

Juvela, M., Montillaud, J., Ysard, N., \& Lunttila, T. 2013, A\&A, 556, A63

Juvela, M., Ristorcelli, I., Pelkonen, V., et al. 2011, A\&A, 527, A111

Kackley, R., Scott, D., Chapin, E., \& Friberg, P. 2010, Proc. SPIE, 7740, $77401 Z$

Kauffmann, J., \& Bertoldi, F. 2004, arXiv:astro-ph/0402021

Kelly, B. C., Shetty, R., Stutz, A. M., et al. 2012, ApJ, 752, 55

Kramer, C., Richer, J., Mookerjea, B., Alves, J., \& Lada, C. 2003, A\&A, 399, 1073
Kuan, Y.-J., Mehringer, D. M., \& Snyder, L. E. 1996, ApJ, 459, 619

Kwon, W., Looney, L. W., Mundy, L. G., Chiang, H.-F., \& Kemball, A. J. 2009, ApJ, 696, 841

Lada, C. J. 1987, in IAU Symp. 115, Star Forming Regions, ed. M. Peimbert \& J. Jugaku (Dordrecht: Reidel), 1

Launhardt, R., Stutz, A. M., Schmiedeke, A., et al. 2013, A\&A, 551, A98

Leroy, A. K., Bolatto, A., Bot, C., et al. 2009, ApJ, 702, 352

Lis, D. C., Serabyn, E., Keene, J., et al. 1998, ApJ, 509, 299

Mathis, J. S. 1990, ARA\&A, 28, 37

Matzner, C. D., \& McKee, C. F. 2000, ApJ, 545, 364

Moffat, A. F. J., Jackson, P. D., \& Fitzgerald, M. P. 1979, A\&AS, 38, 197

Mookerjea, B., Ghosh, S. K., Rengarajan, T. N., Tandon, S. N., \& Verma, R. P. 2000, AJ, 120, 1954

Motte, F., Zavagno, A., Bontemps, S., et al. 2010, A\&A, 518, L77

Mueller, K. E., Shirley, Y. L., Evans, N. J., II., \& Jacobson, H. R. 2002, ApJS, 143,469

Myers, P. C., Dame, T. M., Thaddeus, P., et al. 1986, ApJ, 301, 398

Papadopoulos, P. P., \& Allen, M. L. 2000, ApJ, 537, 631

Paradis, D., Veneziani, M., Noriega-Crespo, A., et al. 2010, A\&A, 520, L8

Pascale, E., Ade, P. A. R., Bock, J. J., et al. 2008, ApJ, 681, 400

Pilbratt, G. L., Riedinger, J. R., Passvogel, T., et al. 2010, A\&A, 518, L1

Pirogov, L. E. 2009, ARep, 53, 1127

Pismis, P., \& Hasse, I. 1976, Ap\&SS, 45, 79

Planck Collaboration, Ade, P. A. R., Aghanim, N., et al. 2011, A\&A, 536, A22

Ricci, L., Testi, L., Natta, A., \& Brooks, K. J. 2010a, A\&A, 521, A66

Ricci, L., Testi, L., Natta, A., Scholz, A., \& de Gregorio-Monsalvo, I. 2012, ApJL, 761, L20

Ricci, L., Testi, L., Natta, A., et al. 2010b, A\&A, 512, A15

Roy, A., Ade, P. A. R., Bock, J. J., et al. 2011, ApJ, 727, 114

Russeil, D., Adami, C., \& Georgelin, Y. M. 2007, A\&A, 470, 161

Russeil, D., Georgelin, Y. M., Georgelin, Y. P., Le Coarer, E., \& Marcelin, M. 1995, A\&AS, 114, 557

Sadavoy, S. I., Di Francesco, J., Johnstone, D., et al. 2013, ApJ, 767, 126

Schnee, S., Enoch, M., Noriega-Crespo, A., et al. 2010, ApJ, 708, 127

Schnee, S., \& Goodman, A. 2005, ApJ, 624, 254

Seaquist, E., Yao, L., Dunne, L., \& Cameron, H. 2004, MNRAS, 349, 1428

Shetty, R., Kauffmann, J., Schnee, S., \& Goodman, A. A. 2009a, ApJ, 696, 676

Shetty, R., Kauffmann, J., Schnee, S., Goodman, A. A., \& Ercolano, B. 2009b, ApJ, 696, 2234

Shirley, Y. L., Evans, N. J., II, Rawlings, J. M. C., \& Gregersen, E. M. 2000, ApJS, 131, 249

Shirley, Y. L., Huard, T. L., Pontoppidan, K. M., et al. 2011, ApJ, 728, 143

Shirley, Y. L., Nordhaus, M. K., Grcevich, J. M., et al. 2005, ApJ, 632, 982

Shu, F. H. 1977, ApJ, 214, 488

Sreenilayam, G. 2012, PhD thesis, Univ. Waterloo

Sridharan, T. K., Beuther, H., Saito, M., Wyrowski, F., \& Schilke, P. 2005, ApJL, 634, L57

Stamatellos, D., Whitworth, A. P., \& Ward-Thompson, D. 2007, MNRAS, 379,1390

Swift, J. J., \& Welch, W. J. 2008, ApJS, 174, 202

Tackenberg, J., Beuther, H., Henning, T., et al. 2012, A\&A, 540, A113

Tafalla, M., Myers, P. C., Caselli, P., Walmsley, C. M., \& Comito, C. 2002, ApJ, 569,815

Thomas, H. S. 2013, Starlink Cookbook 21 (Hilo, HI: Joint Astronomy Centre), http://www.starlink.ac.uk/docs/sc21.htx/sc21.html

Ubach, C., Maddison, S. T., Wright, C. M., et al. 2012, MNRAS, 425, 3137

van der Tak, F. F. S., van Dishoeck, E. F., Evans, N. J., II., \& Blake, G. A 2000, ApJ, 537, 283

van Dishoeck, E. F., \& Black, J. H. 1988, ApJ, 334, 771

Ward, R. L., Wadsley, J., Sills, A., \& Petitclerc, N. 2012, ApJ, 756, 119

Ward-Thompson, D., Motte, F., \& Andre, P. 1999, MNRAS, 305, 143

Ward-Thompson, D., Scott, P. F., Hills, R. E., \& Andre, P. 1994, MNRAS, 268, 276

Whittet, D. C. B. (ed.) 2003, Dust in the Galactic Environment (2nd ed.; Bristol: Institute of Physics (IOP) Publishing)

Wilcock, L. A., Ward-Thompson, D., Kirk, J. M., et al. 2012, MNRAS, 424,716

Williams, S. J., Fuller, G. A., \& Sridharan, T. K. 2005, A\&A, 434, 257

12 http://leejjoon.github.com/pywcsgrid2/ 